\title{
Momentum and position detection in nanoelectromechanical systems beyond Born and Markov approximations
}

\author{
Stefan Walter and Björn Trauzettel \\ Institute for Theoretical Physics and Astrophysics, \\ University of Würzburg, 97074 Würzburg, Germany
}

(Dated: June 8, 2022)

\begin{abstract}
We propose and analyze different schemes to probe the quantum nature of nanoelectromechanical systems (NEMS) by a tunnel junction detector. Using the Keldysh technique, we are able to investigate the dynamics of the combined system for an arbitrary ratio of $e V / \hbar \Omega$, where $V$ is the applied bias of the tunnel junction and $\Omega$ the eigenfrequency of the oscillator. In this sense, we go beyond the Markov approximation of previous works where these parameters were restricted to the regime $e V / \hbar \Omega \gg 1$. Furthermore, we also go beyond the Born approximation because we calculate the finite frequency current noise of the tunnel junction up to fourth order in the tunneling amplitudes.

Interestingly, we discover different ways to probe both position and momentum properties of NEMS. On the one hand, for a non-stationary oscillator, we find a complex finite frequency noise of the tunnel junction. By analyzing the real and the imaginary part of this noise separately, we conclude that a simple tunnel junction detector can probe both position- and momentum-based observables of the non-stationary oscillator. On the other hand, for a stationary oscillator, a more complicated setup based on an Aharonov-Bohm-loop tunnel junction detector is needed. It still allows us to extract position and momentum information of the oscillator. For this type of detector, we analyze for the first time what happens if the energy scales $\mathrm{eV}, \hbar \Omega$, and $k_{B} T$ take arbitrary values with respect to each other where $T$ is the temperature of an external heat bath. Under these circumstances, we show that it is possible to uniquely identify the quantum state of the oscillator by a finite frequency noise measurement.
\end{abstract}

PACS numbers: 85.85.+j, 72.70.+m, 07.50.Hp, 42.50.Lc

\section{INTRODUCTION}

Nanoelectromechanical systems (NEMS) have become a promising playground for probing the quantum behavior of mesoscopic objects, theoretically as well as experimentally 12. The diverse reasons to study NEMS are their vast number of (possible) applications as for instance the measurement of mass, force and position ${ }^{3 / 7}$ with high precision.

Nanomechanical systems being at the boarder of classical to quantum are also being studied from a very fundamental point of view. This includes the observation, measurement and control of quantum states of a mesoscopic mechanical continuous variable system such as a harmonic oscillator. Superconducting qubits electrically coupled to the mechanical system have been successfully used to characterize the mechanical resonator's quantum $\operatorname{state}^{8}$.

Making quantum effects visible in nanomechanical systems calls for ultralow temperatures and low dissipation. The goal of observing the quantum mechanical ground state of a harmonic oscillator requires temperatures $k_{B} T \ll \hbar \Omega$. Recently, this goal has been achieved by using a microwave-frequency mechanical oscillator, with a frequency of $f \approx 6 \mathrm{GHz}$ which allowed cooling to the ground state with conventional cryogenic refrigeration ${ }^{9}$. Further proposals of cooling a nanomechanical resonator coupled to an optical cavity have been proposed $\frac{10 \mid 11}{1}$ and experimentally implemented 12113 .

The theoretical treatment of NEMS widely uses a
Markovian master equation approach $\frac{14}{16}$ with a few exceptions, for instance, the work by Wabnig et al.17 and Rastelli et al ${ }^{[18}$ where a Keldysh perturbation theory has been employed. Here, we also make use of the Keldysh technique because it allows us to treat the non-equilibrium system fully quantum mechanically and, furthermore, to carefully investigate the non-Markovian regime where $e V \ll \hbar \Omega$. Since we are interested in the quantum nature of the oscillator, it is important that the temperature $T$ and the applied bias $V$ of the tunnel junction are not much larger than the eigenfrequency $\Omega$ of the oscillator. Otherwise, the oscillator would be heated and low energy properties inaccessible.

The article is organized as follows. Our key results are summarized in Sec. III. In Sec. III. we introduce the generic model. This is followed by an introduction of the formalism we use in Sec. IV with subsections focusing on the fermionic reservoir and on the oscillator dynamics. The main part of this article is presented in Sec. $\mathrm{V}$, where we discuss the calculation as well as the results for the finite frequency current noise. Finally, we conclude in Sec. VI.

\section{KEY RESULTS OF THE PAPER}

The motivation of our work is to study an experimentally feasible setup in which the quantum nature of NEMS can be probed by current noise measurements of a tunnel junction detector. A quantum NEMS can 
be described by a quantum harmonic oscillator which is a continuous variable system characterized by two noncommuting operators $\hat{x}$ and $\hat{p}$. Therefore, it is desirable to have a detector at hand that can measure expectation values with respect to $\hat{x}$-dependent observables, $\hat{p}$ dependent observables, as well as observables that depend on both $\hat{x}$ and $\hat{p}$.

In Ref. 16, Doiron et al. have proposed a setup which could be used for position and momentum detection of NEMS. This setup consists of two tunnel junctions forming an Aharonov-Bohm (AB) loop. There, it is possible to tune the relative phase between the tunnel amplitudes (where one depends on $\hat{x}$ and the other one not) via a magnetic flux penetrating the $\mathrm{AB}$ loop, see Fig. 1 for the schematic setup. In such a setup, the symmetrized current noise

$$
S_{\mathrm{sym}}(\omega)=\frac{1}{2} \int d t e^{i \omega t}\langle\{\Delta \hat{I}(t), \Delta \hat{I}(0)\}\rangle
$$

(with the current fluctuation operator $\Delta \hat{I}(t)=\hat{I}(t)-$ $\langle\hat{I}\rangle)$ of the tunnel junction detector can either contain information on the oscillator's position spectrum

$$
S_{x}(\omega)=\frac{1}{2} \int d t e^{i \omega t}\langle\{\hat{x}(t), \hat{x}(0)\}\rangle,
$$

then $S_{\mathrm{sym}}(\omega) \sim S_{x}(\omega)$, or the oscillator's momentum spectrum

$$
S_{p}(\omega)=\frac{1}{2} \int d t e^{i \omega t}\langle\{\hat{p}(t), \hat{p}(0)\}\rangle,
$$

then $S_{\text {sym }}(\omega) \sim S_{p}(\omega)$, see also Eq. (55) below. The current noise $S_{\mathrm{sym}}(\omega)$ can also contain information on both, the position and the momentum of the oscillator. The former case has been coined $x$-detector and the latter case $p$-detector. We note that $S_{\mathrm{sym}}(\omega), S_{x}(\omega)$, and $S_{p}(\omega)$ are properly defined above in Eqs. (1)-(3) for a stationary problem. In the non-stationary case, which is also subject of discussion in this work, these quantities do not only depend on a single frequency $\omega$ but on one frequency argument and one time argument instead, see Eq. (39).

To be more specific, in this article, we call an $x$ detector, a detector that allows to measure expectation values of the oscillator's position operator $\hat{x}$, i.e. $\langle\hat{x}\rangle$, $\langle\hat{x} \hat{x}\rangle$, etc.. Similarly, we call a $p$-detector, a detector that allows to measure expectation values of $\hat{p}$, the oscillator's momentum operator, i.e. $\langle\hat{p}\rangle,\langle\hat{p} \hat{p}\rangle$, etc.. In Ref. 16, switching from the $x$-detector to the $p$-detector is then accomplished by tuning the relative phase between the tunnel amplitudes. The main difficulty of this setup is the need of long coherence times and length in the $\mathrm{AB}$ loop to make the switching possible. Here, we show that the AB setup can be avoided. We find that the current noise of the coupled oscillator-junction system with one tunnel junction only, already can be used for momentum detection due to the complex nature of the current noise when the oscillator is in a non-stationary state. This is the first key result of our work, specified and intensively discussed in Sec. VB2 below.

We further investigate the current noise stemming from a stationary oscillator up to fourth order in the tunneling amplitudes, thereby going beyond the Born approximation. Most importantly, we extend previous results of Ref. 16 to the non-Markovian regime without any restrictions on the relative magnitude of the energy scales $e V, \hbar \Omega$, and $k_{B} T$. We show that peaks in the finite frequency current noise at $\omega= \pm \Omega$ (both for the $x$ detector and the $p$-detector) are a fourth order effect. In the Markovian regime, the peaks in the position detector signal are always much larger than the ones in the momentum detector signal. This is different in the non-Markovian regime. There, we even find a larger signal for the momentum detector compared to the position detector, clearly demonstrating that the non-Markovian regime is the preferred regime to operate the momentum detector. The detailed understanding of the $x$-detector and the $p$-detector developed in this article allows us to uniquely identify the quantum state of the oscillator by a finite frequency noise measurement. This is the second key result of our work, specified and intensively discussed in Sec. VC4 and Sec. VC5 below.

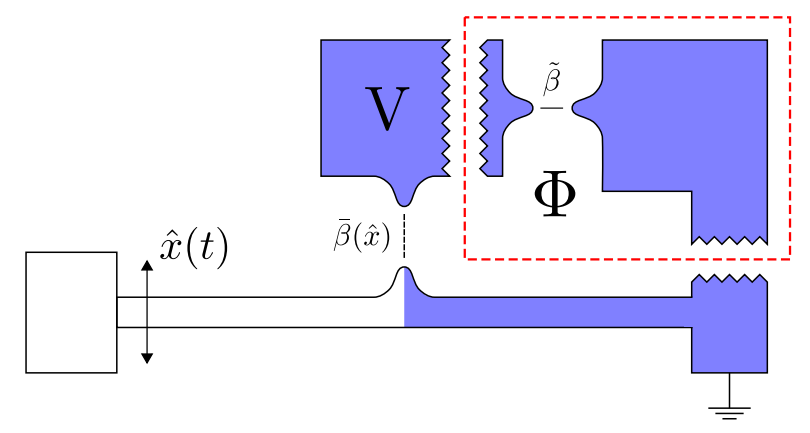

FIG. 1. Schematic setup for the realization of a position detector which can be extended by the parts enclosed in the red dashed box to a momentum detector. The total tunnel amplitude in the case of the position detector is given as $\beta=$ $\bar{\beta}(\hat{x})=t_{0}+t_{1} \hat{x}$ and in the case of the momentum detector as $\beta=\bar{\beta}(\hat{x})+\tilde{\beta}=t_{0}+t_{1} e^{i \eta} \hat{x}$, where the relative phase $\eta$ between $t_{0}$ and $t_{1}$ can be tuned via a magnetic flux $\Phi$ penetrating the AB loop. If the oscillator is in a non-stationary state, already the parts without the elements in the red dashed box serve as a position as well as a momentum detector.

\section{MODEL}

The system we consider consists of a nanomechanical harmonic oscillator coupled to a biased tunnel junction. In Ref. 19 an experimental realization is shown, where electrons can tunnel from an atomic point contact (APC) onto a conducting oscillator. The coupled system is described by the following Hamiltonian

$$
\hat{H}=\hat{H}_{\text {osc }}+\hat{H}_{\text {res }}+\hat{H}_{\text {tun }},
$$


with

$$
\begin{aligned}
& \hat{H}_{\mathrm{osc}}=\frac{\hat{p}^{2}}{2 m}+\frac{1}{2} m \Omega^{2} \hat{x}^{2} \\
& \hat{H}_{\mathrm{res}}=\sum_{l, r} \varepsilon_{l} \hat{c}_{l}^{\dagger} \hat{c}_{l}+\varepsilon_{r} \hat{c}_{r}^{\dagger} \hat{c}_{r},
\end{aligned}
$$

where $\hat{H}_{\text {osc }}$ describes the oscillator with $\hat{x}$ and $\hat{p}$ being the position and momentum operator of the oscillator with mass $m$ and frequency $\Omega$, respectively. $\hat{H}_{\text {res }}$ contains the fermionic reservoirs of the left and right contacts. The oscillator couples to the tunnel junction via the tunneling Hamiltonian

$$
\hat{H}_{\mathrm{tun}}=\sum_{l, r} \beta \hat{c}_{l}^{\dagger} \hat{c}_{r}+\text { h.c. }
$$

Here $\hat{c}_{i}\left(\hat{c}_{i}^{\dagger}\right)$ annihilates (creates) an electron in reservoir $i=l, r$. Motivated by the experimental setup in Ref. 19, we take the oscillator to act as one of the fermionic reservoirs. Therefore, the tunneling gap depends on the position of the oscillator, modifying the tunneling amplitude of the APC. For small oscillator displacements $x$, we assume linear coupling of the oscillator to the tunnel junction with a tunnel amplitude $\beta_{1}$. Hence we obtain $\beta=\left[\beta_{0}+\beta_{1} \hat{x}\right]$, with $\beta_{0}$ being the bare tunneling amplitude. Here, we allow for complex tunnel amplitudes $\beta_{0}$ and $\beta_{1}$ as previously discussed in Refs. 16 and 20. With $\eta$ we denote the relative phase between the tunnel amplitudes, i.e. we write $\beta_{0}=t_{0}$ and $\beta_{1}=t_{1} e^{i \eta}$ where $t_{0}, t_{1} \in \Re$. A possible experimental realization of the finite and tunable phase $\eta$ is discussed in Ref. 16. As a consequence this phase $\eta$ gives rise to the possibility to detect the oscillator's momentum expectation value $\left\langle\hat{p}^{2}\right\rangle$, present in the current noise.

\section{GREEN'S FUNCTIONS OF THE FERMIONIC RESERVOIR AND OF THE OSCILLATOR USING THE KELDYSH TECHNIQUE}

The true non-equilibrium, non-Markovian quantum behavior of the coupled system is the subject of our interest. Therefore, we make use of the Keldysh formalism ${ }^{2122}$ for our calculation. The quantities being accessible in the experiment are for instance the tunnel current and the current-current correlator, the noise of the tunnel junction. These will also be the main objects of interest in this article. We employ a perturbation theory in the tunnel Hamiltonian $\hat{H}_{\text {tun }}$ and calculate the noise up to fourth order in the tunneling.

The current operator is given by $\hat{I}=-e \dot{\hat{N}}_{l}$, where $\hat{N}_{l}=\hat{c}_{l}^{\dagger} \hat{c}_{l}$ counts electrons in the left reservoir. We then write the current operator as

$$
\hat{I}=e\left[\hat{j}_{0}+\hat{x} \hat{j}_{1}\right]
$$

and similarly

$$
\hat{H}_{\text {tun }}=\hat{h}_{0}+\hat{x} \hat{h}_{1}
$$

with $\hat{j}_{i}=i\left[\hat{\mathcal{T}}_{i}-\hat{\mathcal{T}}_{i}^{\dagger}\right]$ and $\hat{h}_{i}=\hat{\mathcal{T}}_{i}+\hat{\mathcal{T}}_{i}^{\dagger}$. The operator $\mathcal{T}_{i}$ is given by $\mathcal{T}_{i}=\sum_{l, r} \beta_{i} \hat{c}_{l}^{\dagger} \hat{c}_{r}$ with $i \in\{0,1\}$.

\section{A. Reservoirs Green's functions}

The fermionic Green's functions of the left and right reservoirs (free electron gas) $G_{l, r}$, are given on the Keldysh contour $C$ by $G_{l, r}\left(t, t^{\prime}\right)=-i\left\langle T_{c} \hat{c}_{l, r}(t) \hat{c}_{l, r}^{\dagger}\left(t^{\prime}\right)\right\rangle$. $T_{c}$ denotes the time ordering operator on the Keldysh contour, placing times lying further along the contour to the left. Figure 2 shows the Keldysh contour $C$. The contour consists of a lower branch, $C_{-}$on which time evolves in forward direction and of an upper branch, $C_{+}$where time evolves in backward direction. Switching from times lying on the contour to real times is done by analytic continuation. The Keldysh Green's functions $G_{l, r}\left(t, t^{\prime}\right)$ can then be represented by a matrix. A Fourier transformation leads to the following Green's functions

$$
\begin{aligned}
G_{l, r}(\omega) & =\left(\begin{array}{cc}
G_{l, r}^{--}(\omega) & G_{l, r}^{-+}(\omega) \\
G_{l, r}^{+-}(\omega) & G_{l, r}^{++}(\omega)
\end{array}\right)= \\
& =2 \pi i \rho_{0}\left(\begin{array}{cc}
n_{l, r}(\omega)-1 / 2 & n_{l, r}(\omega) \\
n_{l, r}(\omega)-1 & n_{l, r}(\omega)-1 / 2
\end{array}\right) .
\end{aligned}
$$

Here we made use of time translation invariance and assumed a constant density of states in the left and right reservoir $\rho_{l}=\rho_{r}=\rho_{0}$. The applied finite bias $\mu_{r}-\mu_{l}=e V$ is included in the Fermi distribution functions $n_{l}=n(\omega-e V / 2)=\left[\exp (\beta(\omega-e V / 2)+1]^{-1}\right.$ and $n_{r}=n(\omega+e V / 2)=\left[\exp (\beta(\omega+e V / 2)+1]^{-1}\right.$. The inverse temperature of electrons in the reservoirs is $\beta=$ $1 / k_{B} T$ and we use units where $\hbar=1$.

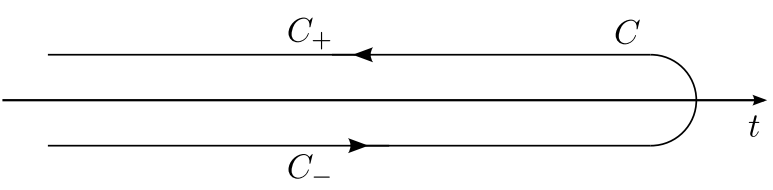

FIG. 2. Keldysh contour $C$ with the lower branch $C_{-}$and the upper branch $C_{+}$.

\section{B. The oscillator}

Since the oscillator modulates the tunneling of electrons and therefore has impact on the measured average current and current-current correlator, it is important to understand the significance of the oscillator's state. We distinguish between an oscillator in a stationary state and 
one in a non-stationary state. We justify this differentiation by arguing that for short times after the measurement, the oscillator will certainly be non-stationary. The dominating timescale here is the one given by the oscillator itself, $1 / \Omega$, which has to be compared to times scales on which the damping of the oscillator due to the tunnel junction and the external heat bath happens. In the nonstationary case, we cannot make use of time translation invariance in the oscillator's correlation function $D\left(t, t^{\prime}\right)$. For longer times however, the assumption of stationarity is justified since the oscillator can equilibrate with the environment and reach a steady state. The oscillator's correlation function now only depends on the time difference $t-t^{\prime}$.

We work in the following with the oscillator operators given in the Heisenberg picture as $\hat{x}(t)=\hat{x} \cos (\Omega t)+$ $\hat{p} /(m \Omega) \sin (\Omega t)$ and $\hat{p}(t)=\hat{p} \cos (\Omega t)-\hat{x}(m \Omega) \sin (\Omega t)$. We also define the aforementioned oscillator correlation function $D\left(t, t^{\prime}\right)$ in Keldysh space as

$$
D\left(t, t^{\prime}\right)=-i\left\langle T_{c} \hat{x}(t) \hat{x}\left(t^{\prime}\right)\right\rangle .
$$

When we later investigate the second order noise we consider both the stationary situation and the nonstationary situation. The following relation then is a very useful one

$$
\hat{x}\left(t+t^{\prime}\right)=\hat{x}\left(t^{\prime}\right) \cos (\Omega t)+\frac{\hat{p}\left(t^{\prime}\right)}{m \Omega} \sin (\Omega t) .
$$

For calculations up to second order, we look at the influence of stationary and non-stationary oscillator states on the current noise, in fourth order we restrict ourselves to the stationary case. Hence, we are interested in a clear definition of the oscillator's correlation functions and spectral functions in the stationary case, which will be addressed now.

\section{Oscillator correlation functions in the stationary case}

Considering the stationary case, we give useful expressions for the oscillator's correlation functions which later allow us to identify the oscillator's power spectrum in $x$ denoted by $S_{x}(\omega)$ and in $p$ denoted by $S_{p}(\omega)$. From Eq. 11 the correlation function where $t \in C_{+}$and $t^{\prime} \in C_{-}$is given by

$$
\begin{aligned}
& i D^{+-}\left(t, t^{\prime}\right)=\left\langle\hat{x}(t) \hat{x}\left(t^{\prime}\right)\right\rangle= \\
& =\frac{1}{2}\left\langle\bar{x}_{+}^{2} \cos \left(\Omega\left(t-t^{\prime}\right)\right)+\frac{[\hat{p}, \hat{x}]}{m \Omega} \sin \left(\Omega\left(t-t^{\prime}\right)\right)+\right. \\
& \left.+\bar{x}_{-}^{2} \cos \left(\Omega\left(t+t^{\prime}\right)\right)+\frac{\{\hat{p}, \hat{x}\}}{m \Omega} \sin \left(\Omega\left(t+t^{\prime}\right)\right)\right\rangle
\end{aligned}
$$

and similar for $t \in C_{-}$and $t^{\prime} \in C_{+}$

$$
\begin{aligned}
& i D^{-+}\left(t, t^{\prime}\right)=\left\langle\hat{x}\left(t^{\prime}\right) \hat{x}(t)\right\rangle= \\
& =\frac{1}{2}\left\langle\bar{x}_{+}^{2} \cos \left(\Omega\left(t-t^{\prime}\right)\right)-\frac{[\hat{p}, \hat{x}]}{m \Omega} \sin \left(\Omega\left(t-t^{\prime}\right)\right)+\right. \\
& \left.+\bar{x}_{-}^{2} \cos \left(\Omega\left(t+t^{\prime}\right)\right)+\frac{\{\hat{p}, \hat{x}\}}{m \Omega} \sin \left(\Omega\left(t+t^{\prime}\right)\right)\right\rangle,
\end{aligned}
$$

where we defined

$$
\bar{x}_{ \pm}^{2}=\hat{x}^{2} \pm \frac{\hat{p}^{2}}{m^{2} \Omega^{2}},
$$

and $[\cdot, \cdot]$ denotes the commutator and $\{\cdot, \cdot\}$ the anticommutator. Since, here we deal with the stationary case, the expectation values $\left\langle\bar{x}_{-}^{2}\right\rangle$ and $\langle\{p, x\}\rangle$ appearing as prefactors of functions depending on $t+t^{\prime}$ equal zero which one can easily check by using any stationary state, e.g. number-states. As one would expect, the correlation function now is a function of the time difference $t-t^{\prime}$ only. The Fourier transform of the correlation functions then yields

$$
\begin{aligned}
i D^{+-}(\omega) & =\frac{1}{2}\left\langle\bar{x}_{+}^{2} \mathcal{R}_{\gamma}^{+}(\omega, \Omega)+\frac{i[\hat{p}, \hat{x}]}{m \Omega} \mathcal{R}_{\gamma}^{-}(\omega, \Omega)\right\rangle, \\
i D^{-+}(\omega) & =\frac{1}{2}\left\langle\bar{x}_{+}^{2} \mathcal{R}_{\gamma}^{+}(\omega, \Omega)-\frac{i[\hat{p}, \hat{x}]}{m \Omega} \mathcal{R}_{\gamma}^{-}(\omega, \Omega)\right\rangle .
\end{aligned}
$$

Additionally, we introduce the two momentum correlation functions $i P^{+-}\left(t, t^{\prime}\right)=\left\langle\hat{p}(t) \hat{p}\left(t^{\prime}\right)\right\rangle$ and $i P^{-+}\left(t, t^{\prime}\right)=$ $\left\langle\hat{p}\left(t^{\prime}\right) \hat{p}(t)\right\rangle$. The same arguments as for $i D^{ \pm \mp}\left(t, t^{\prime}\right)$ lead here to the following Fourier transforms

$$
\begin{aligned}
& i P^{+-}(\omega)=\frac{1}{2}\left\langle\bar{p}_{+}^{2} \mathcal{R}_{\gamma}^{+}(\omega, \Omega)+m \Omega i[\hat{p}, \hat{x}] \mathcal{R}_{\gamma}^{-}(\omega, \Omega)\right\rangle, \\
& i P^{-+}(\omega)=\frac{1}{2}\left\langle\bar{p}_{+}^{2} \mathcal{R}_{\gamma}^{+}(\omega, \Omega)-m \Omega i[\hat{p}, \hat{x}] \mathcal{R}_{\gamma}^{-}(\omega, \Omega)\right\rangle,
\end{aligned}
$$

where similar to above

$$
\bar{p}_{ \pm}^{2}=m^{2} \Omega^{2} \hat{x}^{2} \pm \hat{p}^{2} .
$$

We introduce the functions $\mathcal{R}_{\gamma}^{ \pm}(\omega, \Omega)$ as

$$
\begin{aligned}
& \mathcal{R}_{\gamma \rightarrow 0}^{+}(\omega, \Omega)=\pi[\delta(\omega+\Omega)+\delta(\omega-\Omega)] \\
& \mathcal{R}_{\gamma \rightarrow 0}^{-}(\omega, \Omega)=\pi[\delta(\omega-\Omega)-\delta(\omega+\Omega)]
\end{aligned}
$$

The coupling of the oscillator to two environments, namely an external heat bath and the tunnel junction, being at the temperatures $T_{\text {env }}$ and $k_{B} T_{\text {junc }}=e V / 2$ respectively, introduces a damping of the oscillator with damping coefficients $\gamma_{0}$ and $\gamma_{+}$respectively. The oscillator dynamics due to the coupling to the tunnel junction can be calculated by solving a Dyson equation for the oscillator correlation function $D\left(t, t^{\prime}\right)$ where the selfenergy is taken to lowest non-vanishing order in the tunnel Hamiltonian, i.e. $\Sigma\left(t, t^{\prime}\right)=-i\left\langle T_{c} \hat{h}_{1}(t) \hat{h}_{1}\left(t^{\prime}\right)\right\rangle$. Using the Keldysh technique as done in Refs. 17 and 24 the oscillator dynamics and the damping coefficient $\gamma_{+}=\pi \rho_{0}^{2} t_{1}^{2} / m$ can be calculated. The coupling to the external heat bath can be added phenomenologically, or particularly as an interaction with a bath of harmonic oscillators. The total damping then follows as $\gamma_{\text {tot }}=\gamma_{0}+\gamma_{+}$. We can assign an effective temperature $T_{\text {eff }}$ to the oscillator with $\gamma_{\text {tot }} T_{\text {eff }}=\gamma_{+} T_{\text {junc }}+\gamma_{0} T_{\text {env }}$. 
This leads to the general case for $\mathcal{R}_{\gamma}^{ \pm}$by replacing the $\delta$-functions in Eqs. 2122 by a Lorentzian, where we include both sources of damping and an oscillator frequency $\Omega \rightarrow \sqrt{\Omega^{2}-\gamma^{2}}$. For this damped case we can write for $\mathcal{R}_{\gamma}^{+}(\omega, \Omega)$ and $\mathcal{R}_{\gamma}^{-}(\omega, \Omega)$

$$
\begin{aligned}
& \mathcal{R}_{\gamma}^{+}(\omega, \Omega)=\frac{2 \gamma_{\mathrm{tot}}\left(\omega^{2}+\Omega^{2}\right)}{4 \gamma_{\mathrm{tot}}^{2} \omega^{2}+\left(\omega^{2}-\Omega^{2}\right)^{2}}, \\
& \mathcal{R}_{\gamma}^{-}(\omega, \Omega)=\frac{4 \gamma_{\mathrm{tot}} \omega \sqrt{\Omega^{2}-\gamma_{\mathrm{tot}}^{2}}}{4 \gamma_{\mathrm{tot}}^{2} \omega^{2}+\left(\omega^{2}-\Omega^{2}\right)^{2}} .
\end{aligned}
$$

We want to stress that we have not made any assumption on the initial time as e.g. $t^{\prime}=0$. With the above made definitions, the following relation (which has to hold for any bosonic correlation function in Keldysh space)

$$
D^{i j}(\omega)=D^{j i}(-\omega)
$$

can easily be verified, here $i$ and $j$ are Keldysh indices. This concludes our discussion on the oscillator correlation functions. We now turn to the spectral functions $S_{x}(\omega)$ and $S_{p}(\omega)$.

\section{The oscillator's spectra in the stationary case}

The symmetrized power spectrum, in general defined as $\frac{1}{2} \int d t e^{i \omega t}\left\langle\left\{\hat{\Upsilon}(t), \hat{\Upsilon}\left(t^{\prime}\right)\right\}\right\rangle$ of the oscillator quantities $\hat{\Upsilon}=\hat{x}, \hat{p}$ is an observable that can be measured by e.g. current noise measurements (as discussed below). Both $S_{x}(\omega)$ and $S_{p}(\omega)$ can be measured through the current noise $S_{\text {sym }}(\omega)$. The expressions for these power spectra are given by

$$
\begin{aligned}
S_{x}(\omega) & =\frac{1}{2} \int d t e^{i \omega t}\left\langle\left\{\hat{x}(t), \hat{x}\left(t^{\prime}\right)\right\}\right\rangle= \\
& =\frac{1}{2} \int d t e^{i \omega t} i\left[D^{+-}\left(t, t^{\prime}\right)+D^{-+}\left(t, t^{\prime}\right)\right]= \\
& =\frac{1}{2} i\left[D^{+-}(\omega)+D^{-+}(\omega)\right]= \\
& =\frac{1}{2}\left\langle\bar{x}^{2}\right\rangle \mathcal{R}_{\gamma}^{+}(\omega, \Omega),
\end{aligned}
$$

and

$$
\begin{aligned}
S_{p}(\omega) & =\frac{1}{2} \int d t e^{i \omega t}\left\langle\left\{\hat{p}(t), \hat{p}\left(t^{\prime}\right)\right\}\right\rangle= \\
& =\frac{1}{2} \int d t e^{i \omega t} i\left[P^{+-}\left(t, t^{\prime}\right)+P^{-+}\left(t, t^{\prime}\right)\right]= \\
& =\frac{1}{2} i\left[P^{+-}(\omega)+P^{-+}(\omega)\right]= \\
& =\frac{1}{2}\left\langle\bar{p}^{2}\right\rangle \mathcal{R}_{\gamma}^{+}(\omega, \Omega) .
\end{aligned}
$$

The momentum and position spectrum are related via the relation

$$
S_{p}(\omega)=m^{2} \Omega^{2} S_{x}(\omega)
$$

We can also write down the spectra using the Keldysh Green's function $D^{K}(\omega)=D^{+-}(\omega)+D^{-+}(\omega)$ which yields

$$
\begin{aligned}
S_{x}(\omega) & =\frac{1}{2} i D^{K}(\omega), \\
S_{p}(\omega) & =\frac{1}{2} m^{2} \Omega^{2} i D^{K}(\omega) .
\end{aligned}
$$

To further simplify the notation, we introduce

$$
\begin{aligned}
Q(\omega) & =\frac{i}{2}\left[D^{+-}(\omega)-D^{-+}(\omega)\right]= \\
& =\frac{i}{2}\left[P^{+-}(\omega)-P^{-+}(\omega)\right]= \\
& =\frac{i}{2} \frac{[\hat{p}, \hat{x}]}{m \Omega} \mathcal{R}_{\gamma}^{-}(\omega, \Omega)= \\
& =\frac{1}{2 m \Omega} \mathcal{R}_{\gamma}^{-}(\omega, \Omega),
\end{aligned}
$$

which is used later in the fourth order noise calculation.

\section{CURRENT NOISE CALCULATIONS}

In this section, we cover a variety of aspects when dealing with the current noise. For all different aspects we find expressions for the noise which are valid for an arbitrary $\eta$ and therefore include the $x$-detector as well as the $p$-detector.

The first part is dedicated to the noise in second order perturbation theory, where we furthermore make the distinction between a stationary harmonic oscillator and a non-stationary one. Beside the Markovian regime $(e V \gg \hbar \Omega)$, the Keldysh formalism also allows us to investigate the non-Markovian regime $(e V \ll \hbar \Omega)$. The main results in this section are that the current noise for a non-stationary oscillator can in principle be complex. In this case, a detectable complex noise would allow for a nearly complete determination of the oscillators covariance matrix $\sigma_{i j}=\operatorname{tr}\left(\hat{\rho}\left\{\hat{\Upsilon}_{i}, \hat{\Upsilon}_{j}\right\} / 2\right)$, where $\hat{\Upsilon}=(\hat{x}, \hat{p})^{T}$. The covariance matrix allows for a complete description of the oscillator's quantum state.

For the stationary oscillator we recover a noise that is real and in accordance with the Wiener-Khinchin theorem. This noise is the well know noise of a bare biased tunnel junction which shows kinks at $|\omega|=|V| 25 \mid 26$, modified by the oscillator leading to kinks at $|\omega|=|V \pm \Omega|$.

In the last part of this section, we deal with the noise up to fourth order in the tunneling amplitudes. Then we restrict ourselves on the stationary case. On the one hand, the fourth order contributions modify the kinks, on the other hand, they give rise to resonances stemming from the oscillator correlation functions 14 16/17.

We now introduce the perturbation theory leading to the noise expression. 


\section{A. Overview}

In general, the expression for the current-current correlator in the Keldysh formalism is given by

$$
\begin{aligned}
S\left(\tau_{3}, \tau_{4}\right) & =\left\langle T_{c} e^{-i \int_{c} d \tilde{\tau} \hat{H}_{\mathrm{tun}}(\tilde{\tau})} \hat{I}\left(\tau_{3}\right) \hat{I}\left(\tau_{4}\right)\right\rangle- \\
& -\left\langle\hat{I}\left(\tau_{3}\right)\right\rangle\left\langle\hat{I}\left(\tau_{4}\right)\right\rangle
\end{aligned}
$$

since we consider only the second and fourth order current noise we write

$$
S\left(\tau_{3}, \tau_{4}\right)=S^{(2)}\left(\tau_{3}, \tau_{4}\right)+S^{(4)}\left(\tau_{3}, \tau_{4}\right),
$$

where

$$
S^{(2)}\left(\tau_{3}, \tau_{4}\right)=\left\langle T_{c} \hat{I}\left(\tau_{3}\right) \hat{I}\left(\tau_{4}\right)\right\rangle
$$

and

$$
\begin{aligned}
& S^{(4)}\left(\tau_{3}, \tau_{4}\right)= \\
& =-\frac{1}{2} \int_{c} d \tau_{1} d \tau_{2}\left\langle T_{c} \hat{H}_{\mathrm{tun}}\left(\tau_{1}\right) \hat{H}_{\mathrm{tun}}\left(\tau_{2}\right) \hat{I}\left(\tau_{3}\right) \hat{I}\left(\tau_{4}\right)\right\rangle+ \\
& +\int_{c} d \tau_{1} d \tau_{2}\left\langle T_{c} \hat{H}_{\mathrm{tun}}\left(\tau_{1}\right) \hat{I}\left(\tau_{3}\right)\right\rangle\left\langle T_{c} \hat{H}_{\mathrm{tun}}\left(\tau_{2}\right) \hat{I}\left(\tau_{4}\right)\right\rangle .
\end{aligned}
$$

The general expression for the average current is given by

$$
\langle I(t)\rangle=\left\langle T_{c} e^{-i \int_{c} d \tau \hat{H}_{\mathrm{tun}}(\tau)} \hat{I}(t)\right\rangle .
$$

To second order in the tunneling amplitudes, the average current can be calculated by

$$
\langle I(t)\rangle=-i \int_{c} d \tau\left\langle T_{c} \hat{H}_{\mathrm{tun}}(\tau) \hat{I}(t)\right\rangle
$$

and we obtain

$$
\begin{aligned}
\langle I(t)\rangle & =2 \pi \rho_{0}^{2} e\left\{t_{0}^{2} e V+\right. \\
& +2 \cos (\eta) t_{0} t_{1} e V\langle\hat{x}(t)\rangle+\sin (\eta) t_{0} t_{1} \frac{\langle\hat{p}(t)\rangle}{m}+ \\
& \left.+t_{1}^{2} e V\langle\hat{x}(t) \hat{x}(t)\rangle-\frac{t_{1}^{2}}{2 m \Omega} \sigma^{-}(\Omega, V)\right\},
\end{aligned}
$$

where $\sigma^{-}(\Omega, V)$ is given in Eq. 43. Our result for the average current is in accordance with Ref. 16. The current noise we calculate is always the frequency-dependent (and in the non-stationary case also time-dependent) symmetrized current noise, defined as

$$
S_{\mathrm{sym}}\left(\omega, t^{\prime}\right)=\frac{1}{2} \int d t e^{i \omega t}\left[S^{-+}\left(t, t^{\prime}\right)+S^{+-}\left(t, t^{\prime}\right)\right]
$$

where for $S^{-+}\left(t, t^{\prime}\right), t \in C_{-}$and $t^{\prime} \in C_{+}$and similar for $S^{+-}\left(t, t^{\prime}\right)$, here $C_{-}$and $C_{+}$are the lower and upper branch of the Keldysh contour $C$, respectively, see Fig. 2 .

B. Current noise to second order in the tunneling amplitudes

We now turn to the calculation of the current noise to second order. With the current operator $\hat{I}$ already being first order in the tunneling amplitudes, the current noise in the Born approximation is given by the following expression

$$
S^{(2) i j}\left(t, t^{\prime}\right)=\left\langle T_{c} \hat{I}\left(t+t^{\prime}\right)_{i} \hat{I}\left(t^{\prime}\right)_{j}\right\rangle
$$

where we used a slightly different definition of the current noise. This definition will be useful when examining the non-stationary case. Due to this definition the time dependance on $t^{\prime}$ in the symmetrized current noise is only present in the oscillator's quantum mechanical expectation values.

The general expression for the current noise in Keldysh space reads

$$
\begin{aligned}
S^{(2) i j}\left(t, t^{\prime}\right) & =e^{2}\left[\mathcal{G}_{00}\left(t+t^{\prime}, t^{\prime}\right)+\right. \\
& +\left\langle\hat{x}\left(t^{\prime}\right)\right\rangle \mathcal{G}_{01}\left(t+t^{\prime}, t^{\prime}\right)+ \\
& +\left\langle\hat{x}\left(t+t^{\prime}\right)\right\rangle \mathcal{G}_{10}\left(t+t^{\prime}, t^{\prime}\right)+ \\
& \left.+i D\left(t+t^{\prime}, t^{\prime}\right) \mathcal{G}_{11}\left(t+t^{\prime}, t^{\prime}\right)\right] .
\end{aligned}
$$

where $\mathcal{G}_{i j}\left(t, t^{\prime}\right)$ is given in App. A.

\section{General expression for the current noise}

In this section, we only make use of time translation invariance in the $\mathcal{G}_{i j}\left(t, t^{\prime}\right)$ functions, the oscillator is taken as non-stationary. Details of the calculation can be found in App. B. The final result we obtain for the symmetrized current noise to second order in the tunneling amplitudes reads 


$$
\begin{aligned}
S_{\mathrm{sym}}^{(2)}\left(\omega, t^{\prime}\right) & =2 \pi \rho_{0}^{2} e^{2}\left\{t_{0}^{2} \sigma^{+}(\omega, V)+\right. \\
& +\left\langle\hat{x}\left(t^{\prime}\right)\right\rangle t_{0} t_{1} \cos (\eta)\left[\sigma^{+}(\omega, V)+\frac{1}{2}\left(\sigma^{+}(\omega+\Omega, V)+\sigma^{+}(\omega-\Omega, V)\right)\right]- \\
& -\left\langle\hat{x}\left(t^{\prime}\right)\right\rangle t_{0} t_{1} i \sin (\eta)\left[\sigma^{-}(\omega, V)-\frac{1}{2}\left(\sigma^{-}(\omega+\Omega, V)+\sigma^{-}(\omega-\Omega, V)\right)\right]- \\
& -\left\langle\hat{p}\left(t^{\prime}\right)\right\rangle \frac{t_{0} t_{1}}{2 m \Omega}\left[\sin (\eta)\left[\sigma^{-}(\omega+\Omega, V)-\sigma^{-}(\omega-\Omega, V)\right]-i \cos (\eta)\left[\sigma^{+}(\omega+\Omega, V)-\sigma^{+}(\omega-\Omega, V)\right]\right]+ \\
& +\left\langle\hat{x}\left(t^{\prime}\right) \hat{x}\left(t^{\prime}\right)\right\rangle \frac{t_{1}^{2}}{2}\left[\sigma^{+}(\omega+\Omega, V)+\sigma^{+}(\omega-\Omega, V)\right]-\frac{t_{1}^{2}}{2 m}- \\
& \left.-\left\langle\left\{\hat{x}\left(t^{\prime}\right), \hat{p}\left(t^{\prime}\right)\right\}\right\rangle \frac{i t_{1}^{2}}{4 m \Omega}\left[\sigma^{+}(\omega+\Omega, V)-\sigma^{+}(\omega-\Omega, V)\right]\right\}
\end{aligned}
$$

where we separated the real and imaginary part using the relative phase $\eta$ between the tunneling amplitudes and in addition introduced

$$
\begin{aligned}
\sigma^{ \pm}(\xi, V)= & \frac{e V+\xi}{2} \operatorname{coth}\left(\beta \frac{e V+\xi}{2}\right) \\
& \pm \frac{e V-\xi}{2} \operatorname{coth}\left(\beta \frac{e V-\xi}{2}\right) .
\end{aligned}
$$

We want to note the important aspect of the current noise that it possibly can have a complex valued character which we discuss later. The gained expression is quite lengthly but provides us with the full quantum mechanical non-equilibrium characteristics of the current noise in the Markovian as well as in the non-Markovian regime. We made no assumptions on the state of the oscillator, which now gives us the possibility to identify momentum properties of the nanomechanical resonator using the current noise spectrum $S_{\mathrm{sym}}^{(2)}\left(\omega, t^{\prime}\right)$. In the next section, we discuss this new possibility of a $p$-detector which involves measuring a complex valued current noise.

\section{Complex current noise and the p-detector in the non-stationary case}

The expression in Eq. (42) allows for a comparison with results obtained in Ref. 16 where it was possible with a phase of $\eta=\pi / 2$ to determine the momentum of the oscillator. In Ref. 16 an Aharonov-Bohm setup allows the tuning of the relative phase $\eta$. The full current noise spectrum there is proportional to the position spectrum $S_{x}(\omega)$ which is peaked at $\omega= \pm \Omega$ in the case of $\eta=0$ and in the case of $\eta=\pi / 2$ is proportional to the momentum spectrum $S_{p}(\omega)$ showing peaks at $\omega= \pm \Omega$. This peaked structure of the current noise spectrum is a fourth order effect, as we will see and discuss later when dealing with the fourth order corrections to the current noise.

As one can see from Eq. 42, already the second order current noise allows to determine the expectation value of the oscillator's momentum and in addition to that of the anticommutator $\{\hat{x}, \hat{p}\}$, even if the phase $\eta=0$, i.e. the Aharonov-Bohm setup becomes obsolete in our case. The signature of the oscillator's momentum $\hat{p}$ in our case is however different than the one in Ref. 16. Instead of the peaked structure, we find a kink-like structure which stems from the fact that we deal with second order perturbation theory.

In order to understand how one can use this to identify the momentum, we have to understand the meaning of a complex current noise. As stated in Ref. 27] a complex valued current noise is in principle a measurable quantity. To have a relevant measurable quantity we would have to average the time dependent current noise $S_{\mathrm{sym}}^{(2)}\left(\omega, t^{\prime}\right)$ over the measurement time $\Delta T$. We could do this in the following way

$$
\bar{S}_{\mathrm{sym}}^{(2)}(\omega)=\frac{1}{\Delta T} \int_{-\Delta T / 2}^{\Delta T / 2} d t^{\prime} S_{\mathrm{sym}}^{(2)}\left(\omega, t^{\prime}\right)
$$

Since the time dependance of the current noise is only visible in the expectation values of the oscillator's variables, it is important for the actual measurement to consider the time scales which are involved. If the measurement time $\Delta T$ is less than the time scale of the oscillator $(1 / \Omega)$, the measured time averaged current noise $\bar{S}_{\mathrm{sym}}^{(2)}(\omega)$ will be time-dependent. If however, the oscillator undergoes multiple oscillation cycles during the measurement time, the current noise will be time-independent. In this case we could as well take the oscillator to be in a stationary state. For a damped oscillator the times scales on which the damping happens have to be taken into account as mentioned already in Sec. IVB.

We conclude with remarks on the interesting nonstationary case, where we can also take $\eta=0$ without losing the information on $\left\langle\hat{p}\left(t^{\prime}\right)\right\rangle$ and in addition obtain information on $\left\langle\left\{\hat{x}\left(t^{\prime}\right), \hat{p}\left(t^{\prime}\right)\right\}\right\rangle$. We intend to give an idea of how to access the information on $\left\langle\hat{p}\left(t^{\prime}\right)\right\rangle$ and $\left\langle\left\{\hat{x}\left(t^{\prime}\right), \hat{p}\left(t^{\prime}\right)\right\}\right\rangle$ available through the complex current noise.

The expectation value of $\left\langle\hat{p}\left(t^{\prime}\right)\right\rangle$ with respect to number states or a linear combination of them, will al- 
ways vanish when averaging over time according to Eq. (44). However, this is different for coherent states $|\alpha\rangle=\exp \left(-|\alpha|^{2} / 2\right) \sum_{n=0}^{\infty} \alpha^{n} / n !|n\rangle$, where we can write $\alpha=|\alpha| \exp (i \delta)$ with $|\alpha|$ being the amplitude and $\delta$ the phase of the coherent state, respectively. The time averaged expectation values $\left\langle\hat{p}\left(t^{\prime}\right)\right\rangle$ and $\left\langle\left\{\hat{x}\left(t^{\prime}\right), \hat{p}\left(t^{\prime}\right)\right\}\right\rangle$ with respect to $|\alpha\rangle$ yield

$$
\left\langle\hat{p}\left(t^{\prime}\right)\right\rangle_{a v}=\sqrt{2 m \Omega} \frac{2|\alpha|}{\Omega \Delta T} \sin (\delta) \sin (\Omega \Delta T / 2),
$$

and

$$
\left\langle\left\{\hat{x}\left(t^{\prime}\right), \hat{p}\left(t^{\prime}\right)\right\}\right\rangle_{a v}=\frac{2|\alpha|^{2}}{\Omega \Delta T} \sin (2 \delta) \sin (\Omega \Delta T) .
$$

For short measurement times $\Delta T<1 / \Omega$ we can write

$$
\begin{gathered}
\lim _{\Delta T \rightarrow 0}\left\langle\hat{p}\left(t^{\prime}\right)\right\rangle_{a v}=\sqrt{2 m \Omega}|\alpha| \sin (\delta), \\
\lim _{\Delta T \rightarrow 0}\left\langle\left\{\hat{x}\left(t^{\prime}\right), \hat{p}\left(t^{\prime}\right)\right\}\right\rangle_{a v}=2|\alpha|^{2} \sin (2 \delta) .
\end{gathered}
$$

We sparate the current noise $\bar{S}_{\mathrm{sym}}^{(2)}(\omega)=\bar{S}_{\mathrm{sym}, \mathrm{R}}^{(2)}(\omega)+$ $\bar{S}_{\text {sym,I }}^{(2)}(\omega)$ into real and imaginary part where we observe that the imaginary part $\bar{S}_{\mathrm{sym}, \mathrm{I}}^{(2)}(\omega)$ only contains information on the oscillator's momentum $\left\langle\hat{p}\left(t^{\prime}\right)\right\rangle$ and the anticommutator $\left\langle\left\{\hat{x}\left(t^{\prime}\right), \hat{p}\left(t^{\prime}\right)\right\}\right\rangle$

$$
\begin{aligned}
& \bar{S}_{\mathrm{sym}, \mathrm{I}}^{(2)}(\omega)=2 \pi \rho_{0}^{2} e^{2}\{ \\
& \frac{1}{\sqrt{2 m \Omega}} t_{0} t_{1}|\alpha| \sin (\delta)\left[\sigma^{+}(\omega+\Omega, V)-\sigma^{+}(\omega-\Omega, V)\right]- \\
& \left.-\frac{t_{1}^{2}|\alpha|^{2}}{2 m \Omega} \sin (2 \delta)\left[\sigma^{+}(\omega+\Omega, V)-\sigma^{+}(\omega-\Omega, V)\right]\right\} .
\end{aligned}
$$

The phase $\delta$ of the coherent state now allows for a determination of the oscillator's momentum. For $\delta=\pi / 2$, the signature in the imaginary part of the time averaged noise $\bar{S}_{\mathrm{sym}, \mathrm{I}}^{(2)}(\omega)$ stems only from the oscillator's momentum. The signal in the non-Markovian regime is more pronounced than in the Markovian regime, see Eq. (49.)

\section{Current noise in the stationary case}

Contrary to the non-stationary case we now also assume time translation invariance in the oscillator correlation function, i.e. $D\left(t, t^{\prime}\right)=D\left(t-t^{\prime}\right)$. One can see that the calculation in the stationary case goes along the same lines as in the non-stationary case. The only difference will be that oscillator expectation values are now taken at time $t^{\prime}=0$, i.e. we encounter for instance $\langle\hat{x}(0)\rangle$ instead of $\left\langle\hat{x}\left(t^{\prime}\right)\right\rangle$.

When interpreting the result for the stationary case we have to keep the constrains on oscillator expectation values in mind. These constrains mentioned in Sec. IV B 1 lead to vanishing expectation values of the anticommutator $\langle\{\hat{x}, \hat{p}\}\rangle$ and vanishing expectation values for $\langle\hat{x}\rangle$ and $\langle\hat{p}\rangle$. The current noise to second order is then equivalent to the ones previously obtained in Refs. 17] and 20, cf. Eq. (C.4) in Ref. 20 with $\gamma_{2}=\langle x\rangle=\langle p\rangle=0$.

\section{Current noise to fourth order in the tunneling amplitudes}

We now turn to the investigation of the fourth order current noise. Since the fourth order perturbation theory involves a large amount of terms we use a diagramatic approach. In the case of the fourth order current noise we restrict ourself to the stationary case for simplicity. An overview of all contributing terms in the non-stationary case is given in App. C. In what follows we give a short explanation of the diagramatics. From Eq. 35 it becomes obvious that $S^{(4)}$ contains fermionic expectation values which have the form

$$
\begin{aligned}
& \mathcal{M}_{\mathrm{i}_{1}, \mathrm{i}_{2}, \mathrm{i}_{3}, \mathrm{i}_{4}}\left(\tau_{1}, \tau_{2}, \tau_{3}, \tau_{4}\right)= \\
& =\left\langle T_{c} \hat{h}_{i_{1}}\left(\tau_{1}\right) \hat{h}_{i_{2}}\left(\tau_{2}\right) \hat{j}_{i_{3}}\left(\tau_{3}\right) \hat{j}_{i_{4}}\left(\tau_{4}\right)\right\rangle= \\
& =\left\langle T_{c} \mathcal{T}_{i_{1}}^{\dagger}\left(\tau_{1}\right) \mathcal{T}_{i_{2}}\left(\tau_{2}\right) \mathcal{T}_{i_{3}}\left(\tau_{3}\right) \mathcal{T}_{i_{4}}^{\dagger}\left(\tau_{4}\right)+\right. \\
& +(3 \leftrightarrow 4)-(2 \leftrightarrow 4)+\text { h.c. }\rangle .
\end{aligned}
$$

The index $i_{j}$ determines whether we are dealing with $\beta_{0}$ or $\beta_{1}, i_{j} \in\{0,1\}$. It is only necessary to evaluate the first expectation value in Eq. (50) using Wick's theorem, the other ones follow as indicated by $(3 \leftrightarrow 4),(2 \leftrightarrow 4)$ and hermitian conjugation. This first term leads to

$$
\begin{aligned}
& \left\langle T_{c} \mathcal{T}_{i_{1}}^{\dagger}\left(\tau_{1}\right) \mathcal{T}_{i_{2}}\left(\tau_{2}\right) \mathcal{T}_{i_{3}}\left(\tau_{3}\right) \mathcal{T}_{i_{4}}^{\dagger}\left(\tau_{4}\right)\right\rangle= \\
& =\beta_{i_{1}}^{*} \beta_{i_{2}} \beta_{i_{3}} \beta_{i_{4}}^{*} \times \\
& \times\left[G_{l}\left(\tau_{1}, \tau_{2}\right) G_{l}\left(\tau_{4}, \tau_{3}\right)-G_{l}\left(\tau_{1}, \tau_{3}\right) G_{l}\left(\tau_{4}, \tau_{2}\right)\right] \times \\
& \times\left[G_{r}\left(\tau_{2}, \tau_{1}\right) G_{r}\left(\tau_{3}, \tau_{4}\right)-G_{r}\left(\tau_{3}, \tau_{1}\right) G_{r}\left(\tau_{2}, \tau_{4}\right)\right] .
\end{aligned}
$$

Similar to Ref. 17 we use a diagrammatic representation for the expression in Eq. (50). As an example,we show the diagrams emerging from the expression in Eq. (51) in Fig. 3 and explain the components of the diagrams. Fermionic Green's functions of the reservoirs are represented by solid lines and vertices are depicted by a dot, labeled with a time variable and a Keldysh index indicating on which branch of the Keldysh contour the time lies. An integration over internal times $\tau_{1}$ and $\tau_{2}$ is implicit. In addition we also have to sum over the two internal Keldysh indices $k$ and $l$. We recognize that we have to deal with two types of diagrams: diagrams which consist of one closed fermion loop (diagrams in the lower panel of Fig. 3 and diagrams consisting of two closed fermion loops/bubbles (diagrams in the upper panel of Fig. 3. These two different types of diagrams give very different contributions to the current noise which we will discuss below. We include the oscillator correlation function $D\left(t, t^{\prime}\right)$ in diagrams by a wiggly line connecting two 


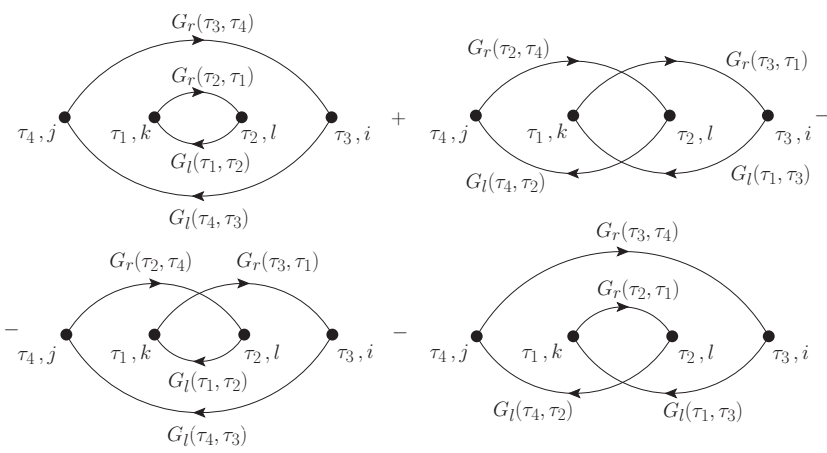

FIG. 3. Diagramatic representation for Eq. (51) where we omitted the factor $\beta_{i_{1}}^{*} \beta_{i_{2}} \beta_{i_{3}} \beta_{i_{4}}^{*}$, note that Eq. (51) only contains fermionic Green's functions.

vertices. In Fig. 4 we give an example of diagrams in frequency space containing one oscillator correlation function. Here, integration over the two internal frequencies $\omega_{1}$ and $\omega_{2}$ as well as summation over the internal Keldysh indices $k$ and $l$ is implied. In frequency space the difference between the closed loop diagrams, $b$ ) in Fig. 4 and the bubble diagrams, $a$ ) in Fig. 4 becomes clear: for the closed loop diagrams, the oscillator line always appears under integration of an internal frequency, whereas for the bubble diagrams, there is no integration over the oscillator line. This is the reason for the different kind of contribution to the current noise of closed loop and bubble diagrams. As we will show below, bubble diagrams lead to peaks in the current noise, whereas closed loop diagrams lead to the afore mentioned kinks in the current noise. We reduce the number of diagrams by only
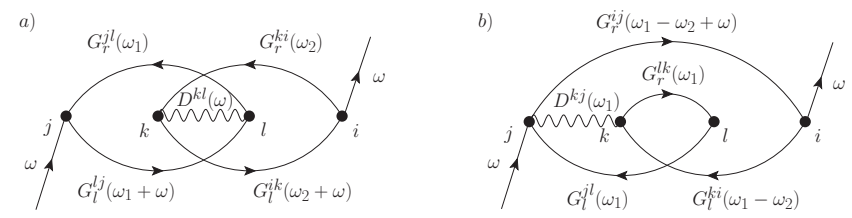

FIG. 4. Examples of diagrams containing one oscillator correlation function $D$. In panel $a$ ) a bubble diagram is depicted where the oscillator line is independent of an internal frequency. Panel $b$ ) shows a closed loop diagram where the oscillator line always appears under integration of an internal frequency.

keeping contributions $\sim t_{0}^{2} t_{1}^{2}$ and $\sim t_{1}^{4}$ which are the only finite contributions in the stationary case. Details are given in App. C. This allows us to write the current noise $S^{(4)}\left(\tau_{3}, \tau_{4}\right)$ for the further analysis as

$$
\begin{aligned}
S^{(4)}\left(\tau_{3}, \tau_{4}\right) & =\hat{S}_{D}^{(4)}\left(\tau_{3}, \tau_{4}\right)+\check{S}_{D}^{(4)}\left(\tau_{3}, \tau_{4}\right)+ \\
& +\hat{S}_{D D}^{(4)}\left(\tau_{3}, \tau_{4}\right)+\check{S}_{D D}^{(4)}\left(\tau_{3}, \tau_{4}\right),
\end{aligned}
$$

where $\hat{S}$ includes the bubble diagrams, $\breve{S}$ includes closed loop diagrams and $D$ indicates the number of oscillator lines in the diagrams. The final result we obtain is valid for an arbitrary relative phase $\eta$ which goes beyond the result obtained by Wabnig et al. in Ref. 17, This fact allows us to study the $p$-detector in fourth order perturbation theory.

$$
\begin{gathered}
\text { 1. Results for } \hat{S}_{D}^{(4)}\left(\tau_{3}, \tau_{4}\right), \check{S}_{D}^{(4)}\left(\tau_{3}, \tau_{4}\right), \hat{S}_{D D}^{(4)}\left(\tau_{3}, \tau_{4}\right) \text { and } \\
\check{S}_{D D}^{(4)}\left(\tau_{3}, \tau_{4}\right)
\end{gathered}
$$

In the following, we sum up the different types of diagrams, bubble type diagrams as well as closed loop diagrams, both then can be integrated exactly.

First we consider all diagrams containing only one oscillator line, these diagrams are all proportional to $t_{0}^{2} t_{1}^{2}$ and depend on $\eta$. We find for the symmetrized frequency dependent current noise

$$
\begin{gathered}
\hat{S}_{\mathrm{sym}, \mathrm{D}}^{(4)}(\omega)=4 \pi^{2} e^{2} \rho_{0}^{4} t_{0}^{2} t_{1}^{2}\{ \\
\cos (\eta)^{2}\left[4 e^{2} V^{2}-4 e V \sigma^{-}(\omega, V) \frac{Q(\omega)}{S_{x}(\omega)}\right] S_{x}(\omega)+ \\
+\sin (\eta)^{2}\left[\omega^{2}-2 \omega \sigma^{+}(\omega, V) \frac{Q(\omega)}{S_{x}(\omega)}\right] S_{x}(\omega)+ \\
+\frac{1}{2}\left[D^{R}(\omega)+D^{A}(\omega)\right] \cos (\eta) \sin (\eta) \times \\
\left.\times\left[4 e V \sigma^{+}(\omega, V)-2 \omega \sigma^{-}(\omega, V)\right]\right\}
\end{gathered}
$$

which is one of the main results of this paper.

In the case of the closed loop diagrams containing one oscillator line, it is also possible to sum up all diagrams and integrate them exactly. The expression for $\check{S}_{\mathrm{sym}, \mathrm{D}}^{(4)}(\omega)$ is rather lengthly, therefore we do not present it here.

We find that the current noise signature of $\check{S}_{\mathrm{sym}, \mathrm{D}}^{(4)}(\omega)$ is of the kink-like structure similar to $S_{\mathrm{sym}, \mathrm{D}}^{(2)}(\omega)$. In addition to the kinks at $|\omega|=|V \pm \Omega|$ coming from $S_{\mathrm{sym}, \mathrm{D}}^{(2)}(\omega)$, $\check{S}_{\mathrm{sym}, \mathrm{D}}^{(4)}(\omega)$ gives rise to extra kinks at $|\omega|=|V|$ and $|\omega|=|\Omega|$. However, these contributions are only minor modifications to the current noise floor $S_{\mathrm{sym}, \mathrm{D}}^{(2)}(\omega)$. Experiments as in Ref. 19 focus on the current noise near the resonance frequency $\omega \approx \Omega$ for which $\hat{S}_{\mathrm{sym}, \mathrm{D}}^{(4)}(\omega)$ is the most important contribution. Therefore, the discussion of our result will focus on the contributions stemming from $\hat{S}_{\text {sym.D }}^{(4)}(\omega)$. These contributions posses a peaked structure, since the oscillator correlation functions $D^{R / A}(\omega)$ and the spectrum $S_{x}(\omega)$ is peaked around $\omega= \pm \Omega$.

The other contributions to the current noise stem from diagrams containing two oscillator lines $D\left(t, t^{\prime}\right)$. These diagrams are all proportional to $\beta_{1} \beta_{1}^{*} \beta_{1} \beta_{1}^{*}=t_{1}^{4}$ and therefore independent of the relative phase $\eta$ between $t_{0}$ and $t_{1}$. Moreover, these current noise contributions are small compared to the ones containing only one oscillator line since $t_{1}^{4} \ll t_{0}^{2} t_{1}^{2}$. We however are mainly interested in 
the possibility to detect the oscillator's momentum which depends on $\eta$, for this reasons and the fact that they are small compared to $\hat{S}_{\mathrm{sym}, \mathrm{D}}^{(4)}(\omega)$ we do not include them in our discussion, nevertheless we state our result which we obtain after summing up the diagrams

$$
\begin{aligned}
& \hat{S}_{D D}^{(4) i j}(\omega)=-\frac{e^{2}}{2 \pi} \int d \omega_{1} \sum_{k, l= \pm}(k l)\{ \\
& D^{k l}\left(\omega_{1}\right) D^{i j}\left(\omega_{1}+\omega\right) \overline{\mathcal{G}}_{11}^{k j}\left(-\omega_{1}\right) \overline{\mathcal{G}}_{11}^{l i}\left(\omega_{1}\right)+ \\
+ & D^{k i}\left(\omega_{1}\right) D^{l j}\left(\omega_{1}+\omega\right) \overline{\mathcal{G}}_{11}^{k j}\left(-\omega_{1}\right) \overline{\mathcal{G}}_{11}^{l i}\left(-\omega_{1}-\omega\right)- \\
- & \left.D^{k i}\left(\omega_{1}\right) D^{l j}\left(-\omega_{1}\right) \mathcal{G}_{11}^{k l}\left(-\omega_{1}\right) \mathcal{G}_{11}^{i j}\left(\omega_{1}+\omega\right)\right\} .
\end{aligned}
$$

The last integration in Eq. (54) can be easily done since the oscillator correlation functions are peaked at $\pm \Omega$. Our result is then in accordance with the one obtained by Wabnig et al. in Ref. 17, where it is has been shown that these contributions to the current noise are peaked at $\omega=-2 \Omega, 0,2 \Omega$ in contrast to the contributions arising from Eq. 53. Similar to $\breve{S}_{D}^{(4)}(\omega), \check{S}_{D D}^{(4)}(\omega)$ is of the kinklike structure and therefore only modifying the current noise floor.

We now address the current noise stemming from $\hat{S}_{\mathrm{sym}, \mathrm{D}}^{(4)}(\omega)$ for arbitrary $\eta$ and also arbitrary system parameters.

2. Current noise in the Markovian and non-Markovian regime for arbitrary $\eta$

In order to compare our result for the momentum detector with Ref. 16, we investigate $\hat{S}_{\mathrm{sym}, \mathrm{D}}^{(4)}(\omega)$ near the resonance $(\omega \approx \Omega)$. We find

$$
\begin{gathered}
\hat{S}_{\mathrm{sym}, \mathrm{D}}^{(4)}(\omega) \approx 4 \pi^{2} e^{2} \rho_{0}^{4} t_{0}^{2} t_{1}^{2}\{ \\
4 e^{2} V^{2} \cos (\eta)^{2}\left[1-\frac{\sigma^{-}(\Omega, V)}{4 e V m \Omega\left\langle\bar{x}^{2}\right\rangle} \sqrt{1-\left(\frac{\gamma_{t o t}}{\Omega}\right)^{2}}\right] S_{x}(\omega)+ \\
+\frac{1}{m^{2}} \sin (\eta)^{2}\left[1-\frac{2 \sigma^{+}(\Omega, V) m}{\left\langle\bar{p}^{2}\right\rangle} \sqrt{1-\left(\frac{\gamma_{t o t}}{\Omega}\right)^{2}}\right] S_{p}(\omega)+ \\
+\cos (\eta) \sin (\eta) \frac{1}{m} \frac{\omega^{2}-\Omega^{2}}{4 \gamma_{t o t}^{2} \Omega^{2}+\left(\omega^{2}-\Omega^{2}\right)^{2}} \times \\
\left.\times\left[4 e V \sigma^{+}(\Omega, V)-2 \Omega \sigma^{-}(\Omega, V)\right]\right\}
\end{gathered}
$$

where $\sigma^{ \pm}(\xi, V)$ is given in Eq. 430 and $S_{x}(\omega)$ and $S_{p}(\omega)$ near resonance are given by

$$
S_{X}(\omega)=\frac{2 \gamma_{\text {tot }}^{2} \Omega^{2}\left\langle X^{2}\right\rangle}{4 \gamma_{\text {tot }}^{2} \Omega^{2}+\left(\omega^{2}-\Omega^{2}\right)^{2}},
$$

with $X=\bar{x}, \bar{p}$. The above expression is valid for the Markovian as well as for the non-Markovian regime. The relevant information about the oscillator can now be gained form the current noise spectrum.
We take two different ways of evaluating the expectation values $\left\langle\bar{x}^{2}\right\rangle$ and $\left\langle\bar{p}^{2}\right\rangle$. In the first one we use number-states which lead to expectation values $\left\langle\bar{x}^{2}\right\rangle_{n}=$ $(2 n+1) / m \Omega$ and $\left\langle\bar{p}^{2}\right\rangle_{n}=(2 n+1) m \Omega$, where $n$ denotes the oscillator's number of quanta.

Since we also could imagine, as already explained in Sec. IV B 1, two equilibrium bathes, the tunnel junction and an external heat bath, to which the oscillator couples, we can assign an effective temperature $T_{\text {eff }}$ to the oscillator which obeys $\gamma_{\text {tot }} T_{\text {eff }}=\gamma_{0} T_{\text {env }}+\gamma_{+} T_{\text {junc }}$, where $\gamma_{\text {tot }}=\gamma_{0}+\gamma_{+}$is the total damping due to coupling to the junction $\left(\gamma_{+}\right)$and an external heat bath $\left(\gamma_{0}\right)$. The external heat bath is at temperature $T_{\text {env }}$ and the junction's temperature is given by $T_{\text {junc }}=e V / 2 k_{B}$. The oscillator's expectation values in this thermal regime are then give by $\left\langle\bar{x}^{2}\right\rangle=2 k_{B} T_{\text {eff }} / m \Omega^{2}$ and $\left\langle\bar{p}^{2}\right\rangle=2 m k_{B} T_{\text {eff }}$.

For both cases, the thermal case and the number-state one, it is convenient scaling the current noise $\hat{S}_{\mathrm{sym}, \mathrm{D}}^{(4)}(\omega)$ with $e I_{0}=2 \pi \rho_{0}^{2} e^{2} t_{0}^{2} \sigma^{+}(\Omega, V)$. We furthermore introduce dimensionless constant $f_{1}, f_{2}, f_{3}, f_{4}$ which are defined in the following way

$$
\begin{aligned}
\gamma_{\text {tot }} & =\frac{\Omega}{f_{1}} \\
\gamma_{+} & =\frac{\gamma_{\text {tot }}}{f_{2}}=\frac{\Omega}{f_{1} f_{2}} \\
e V & =f_{3} \Omega \\
T_{\text {env }} & =f_{4} \frac{e V}{k_{B}} .
\end{aligned}
$$

$f_{1}$ can be interpreted as an overall quality-factor. The ratio $\gamma_{0} / \gamma_{+}=\left(f_{2}-1\right)$ leads for $\left.f_{2} \in\right] 1,2[$ to a stronger coupling to the tunnel junction and for $f_{2}>2$ to a stronger coupling to the external heat bath. The parameter $f_{3}$ distinguishes the non-Markovian $\left.\left.\left(f_{3} \in\right] 0,1\right]\right)$ from the Markovian regime $\left(f_{3} \gg 1\right)$. The last parameter $f_{4}$ quantifies the temperature $T_{\text {env }}$ of the external bath wit respect to the applied bias $V$.

We now compare the signal of the position detector $S_{\mathrm{x}-\mathrm{det}}^{(4)}(\omega)=\hat{S}_{\mathrm{sym}, \mathrm{D}}^{(4)}(\omega ; \eta=0)$ to the signal of the momentum detector $S_{\mathrm{p}-\mathrm{det}}^{(4)}(\omega)=\hat{S}_{\mathrm{sym}, \mathrm{D}}^{(4)}(\omega ; \eta=$ $\pi / 2$ ) and later their dependencies on the parameters $f_{i}$ at resonance $\omega \approx \Omega$. Assuming a high quality resonator we take $\sqrt{1-\gamma_{\text {tot }}^{2} / \Omega^{2}} \approx 1$ in Eq. (55). We call $\mathcal{Q}_{x}=\sigma^{-}(\Omega, V) /\left(4 \mathrm{eVm} \Omega\left\langle\bar{x}^{2}\right\rangle\right)$ quantum corrections to the $x$-detector current noise, arising from the non-vanishing commutator $[\hat{x}, \hat{p}]$, similarly we call $\mathcal{Q}_{p}=\left(2 m \sigma^{+}(\Omega, V)\right) /\left\langle\bar{p}^{2}\right\rangle$ quantum corrections to the $p$-detector current noise. We then find

$$
S_{\mathrm{x}-\mathrm{det}}^{(4)}=4 f_{3}^{2} \frac{\left[1-\mathcal{Q}_{x}\right]}{\left[1-\mathcal{Q}_{p}\right]} S_{\mathrm{p}-\mathrm{det}}^{(4)}
$$

and conclude that in the Markovian regime where $f_{3} \gg 1$ the signal of the position detector is always larger than the signal of the momentum detector. Whereas in the non-Markovian regime we have a stronger signature of 
the noise part showing the momentum signature of the oscillator. In the following, we investigate in more detail the current noise of the $x$ - and $p$-detector.

\section{The $x$-detector}

From Eq. (55) one can see that for $\eta=0 \bmod \pi$ we recover the position detector result as in Refs. 14, 15, and 17, with peaks in the current noise spectrum at $\omega= \pm \Omega$. Since we calculate the symmetrized current-current correlator, the current noise is symmetric in $\omega$. The sign of the signal is given by the sign of $\left[1-\mathcal{Q}_{x}\right]$ which for an oscillator in the thermal regime depends on $f_{2}, f_{3}$ and $f_{4}$, for an oscillator in number-state $n$ it depends on $n$ and $f_{3}$ only. We stick to a thermal resonator, noting that as in Ref. 16 for the $p$-detector, here the quantum corrections $\mathcal{Q}_{x} \sim 1 / f_{3}$ can become large (compared to 1 ) in the non-Markovian regime where $f_{3}<1$, leading to a sign change. The parameter regimes for a negative/positive peak in the current noise are depicted in Fig. 5 as blue/red regions. The change of sign in the

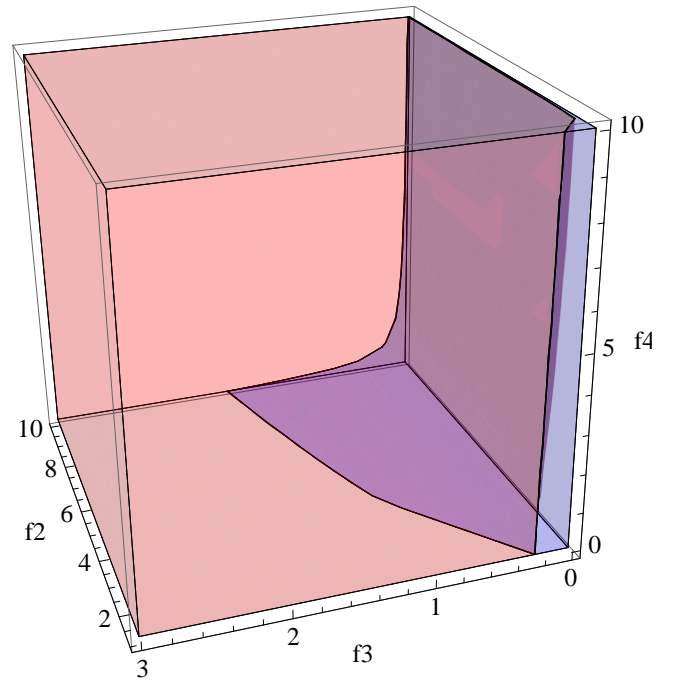

FIG. 5. The current noise peak at $\omega=\Omega$ for the $x$-detector as a function of $f_{2}, f_{3}, f_{4}$, where we took $f_{1} \rightarrow \infty$. The blue region shows the parameter regime where the peak is negative, for parameter combinations lying in the red region, the peak is positive.

signal, depends on the environment temperature $T_{\text {env }}$, the coupling to the environments $f_{2}$ and heavily on the bias voltage and therefore $f_{3}$. Deep in the Markovian regime the sign change is hard to achieve, only if $f_{2}$ is very large and the bath temperature $T_{\text {env }}$ is very low, meaning that heating of the oscillator can be compensated by strongly coupling to a cold environment. In the non-Markovian regime the quantum corrections $\mathcal{Q}_{x}$ can become large more easily and due to the lower signal in the non-Markovian regime for the $x$-detector, the sign change is more pronounced.
Figs. 6 and 7] show the current noise spectrum around $\omega \approx \Omega$ in the Markovian and the non-Markovian regime for different couplings and environment temperatures.

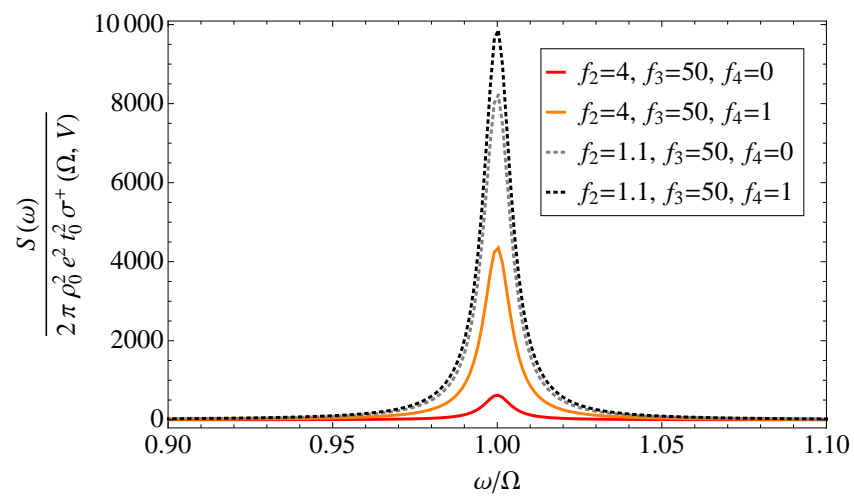

FIG. 6. Noise signal at $\omega=\Omega$ in the Markovian regime for different values of $f_{2}, f_{3}, f_{4}$ and $f_{1}=200$ which shows a peak, due to the presence of the oscillator.

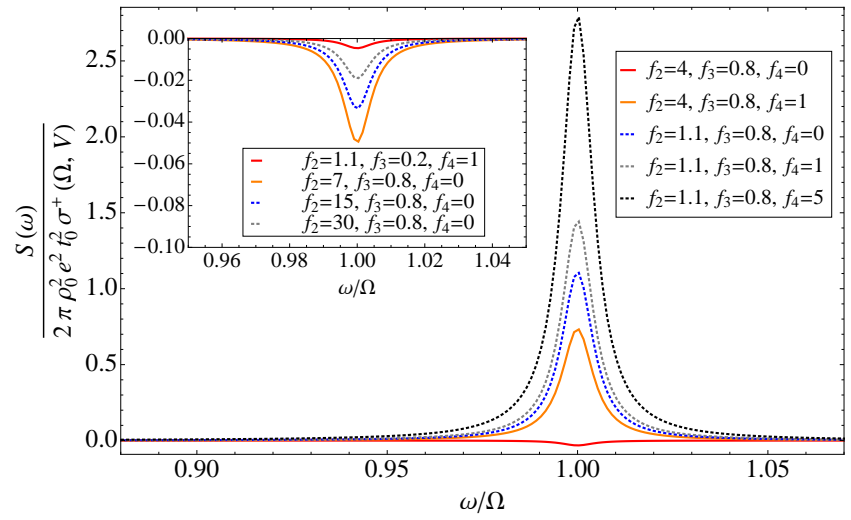

FIG. 7. $x$-detector current noise signal at $\omega=\Omega$ in the non-Markovian regime for different values of $f_{2}, f_{3}, f_{4}$ and $f_{1}=200$. The signal is weaker than in the Markovian regime (compare to Fig. 6). In this regime however, it is possible to see a change of sign of the signal, depending on the parameters $f_{2}, f_{4}$. The inset illustrates this sign change.

\section{The p-detector}

For the cases $\eta=\pi / 2 \bmod \pi$ in Eq. $(55)$, the result of the momentum detector as stated in Ref. 16 are extended to the non-Makovian regime. Due to the fact that the quantum corrections $\mathcal{Q}_{p}$ are in the first place larger than $\mathcal{Q}_{x}$, the peak in the current noise spectrum stemming from the oscillator has a negative sign for $\eta=\pi / 2$. However, it is also possible to change the sign by adjusting the parameters $f_{2}, f_{3}, f_{4}$ on which $\mathcal{Q}_{p}$ depends. In Fig. 8 we depict the regions with a negative sign blue and the ones 
with a positive sign red. Changing the sign of the current noise signal in the $p$-detector case is easier to achieve over a wide range of parameters, as compared to the $x$ detector, even deep in the Markovian regime $\left(f_{3} \gg 1\right)$. Figure 9 shows a summary of the $p$-detector current noise in the Markovian and non-Markovian regime for different parameters $f_{2}, f_{4}$, respectively.

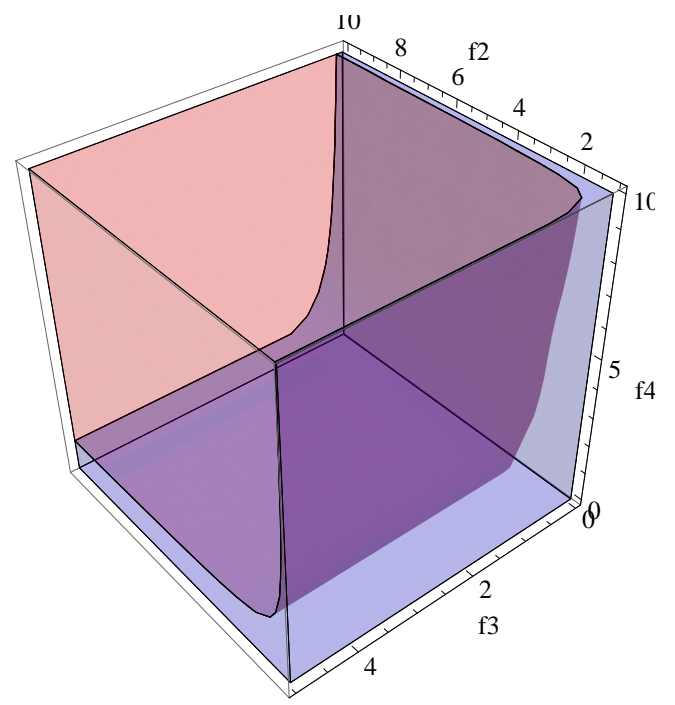

FIG. 8. This figure shows the parameter regimes for the $p$-detector where the current noise peak at $\omega=\Omega$ has a negative sign (blue) and positive sign (red). Compared to the $x$-detector the sign change is possible for a wider range of parameters $f_{2}, f_{3}, f_{4}$; we took $f_{1} \rightarrow \infty$.
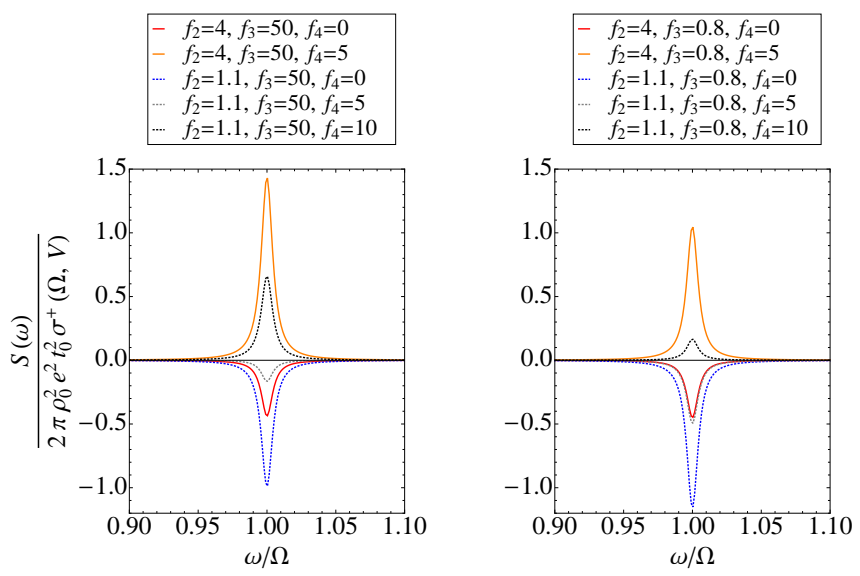

FIG. 9. The left panel shows the $p$-detector current noise in the Markovian regime for various parameters $f_{2}, f_{4}$ and $f_{1}=200$. The right panel shows the current noise in the non-Markovian regime for various parameters $f_{2}, f_{4}$. In both cases we can easily achieve a sign change in the signal.

\section{Detection of number states}

With the above made definitions we can map the occupation number of the oscillator to experimentally adjustable parameters, as for instance the environment temperature $T_{\text {env }}$ and the bias voltage $V$, similar to the approach in Ref. 17. This allows us in general to determine which state $n$ the oscillator is in. This mapping is independent of $\eta$ and depends only on the dimensionless parameters $f_{i}$ in the following way

$$
n=\frac{2 f_{2}\left|f_{3}\right| f_{4}-2\left|f_{3}\right| f_{4}-f_{2}+\left|f_{3}\right|}{2 f_{2}} .
$$

The dependance of $n$ on adjustable parameters is depicted in Fig. 10. In the Markovian regime the oscillator
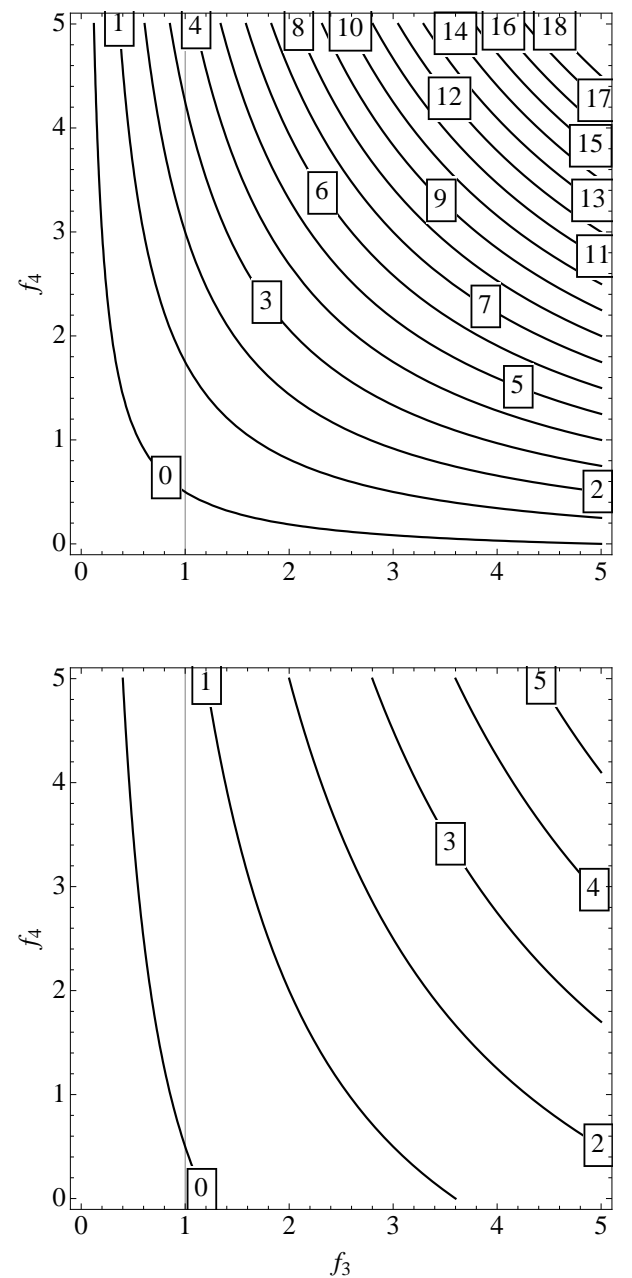

FIG. 10. Number of quanta $n$ on the oscillator in dependence of experimentally adjustable parameters, for dominant coupling to the external heat bath (upper panel, with $f_{2}=5$ ) and dominant coupling to the tunnel junction (lower panel, with $\left.f_{2}=1.2\right)$.

is only in its ground state for low environmental temperatures, since the applied bias voltage is heating the oscillator. In the non-Markovian regime, we can have a 
higher environmental temperature for the oscillator being in its ground state.

\section{CONCLUSION}

We have studied the finite frequency current noise of a tunnel junction coupled to a harmonic oscillator. In our work, we go beyond the Born approximation (because we calculate the noise up to fourth order in the tunneling amplitude) and beyond the Markov approximation (because we do not restrict ourselves to the regime $\mathrm{eV} / \hbar \Omega \gg 1$ ). For a non-stationary oscillator, we have shown that the finite frequency current noise of the detector can be complex. This complex current noise can be used to obtain information about expectation values depending on $\hat{x}$ as well as expectation values depending on $\hat{p}$. The former we call $x$-detector signal and the latter $p$-detector signal.

For the stationary oscillator, the finite frequency cur- rent noise is always real. Then, it is more complicated to get momentum information using a tunnel junction detector. An Aharonov-Bohm-loop setup is needed for this task. We analyze such a setup for the first time in the non-Markovian regime and thereby show how the $x$ - and the $p$-signal can be used to determine the quantum state of the oscillator.

Our analysis is an essential prerequisite to study how the quantum entanglement of NEMS $\overline{28}$ can be measured on the basis of tunnel junction detectors. This very interesting problem will be addressed in future work.

\section{ACKNOWLEDGMENTS}

We thank T. L. Schmidt and K. Børkje for interesting discussions and acknowledge financial support from the DFG.

\section{Appendix A: Details on the fermionic Green's functions}

Expectation values of the fermionic operators $\hat{h}_{i}$ and $\hat{j}_{i}$ can be expressed by the Keldysh Green's functions $G_{l, r}\left(t, t^{\prime}\right)=-i\left\langle T_{c} \hat{c}_{l, r}(t) \hat{c}_{l, r}^{\dagger}\left(t^{\prime}\right)\right\rangle$ as

$$
\begin{aligned}
\left\langle T_{c} \hat{h}_{i}(t) \hat{j}_{j}\left(t^{\prime}\right)\right\rangle & =-i \overline{\mathcal{G}}_{i j}\left(t, t^{\prime}\right)=-i\left[\beta_{i} \beta_{j}^{*} G_{r}\left(t, t^{\prime}\right) G_{l}\left(t^{\prime}, t\right)-\beta_{i}^{*} \beta_{j} G_{r}\left(t^{\prime}, t\right) G_{l}\left(t, t^{\prime}\right)\right] \\
\left\langle T_{c} \hat{h}_{i}(t) \hat{h}_{j}\left(t^{\prime}\right)\right\rangle & =\mathcal{G}_{i j}\left(t, t^{\prime}\right)=\left[\beta_{i} \beta_{j}^{*} G_{r}\left(t, t^{\prime}\right) G_{l}\left(t^{\prime}, t\right)+\beta_{i}^{*} \beta_{j} G_{r}\left(t^{\prime}, t\right) G_{l}\left(t, t^{\prime}\right)\right] \\
\left\langle T_{c} \hat{j}_{i}(t) \hat{j}_{j}\left(t^{\prime}\right)\right\rangle & =\mathcal{G}_{i j}\left(t, t^{\prime}\right)=\left[\beta_{i} \beta_{j}^{*} G_{r}\left(t, t^{\prime}\right) G_{l}\left(t^{\prime}, t\right)+\beta_{i}^{*} \beta_{j} G_{r}\left(t^{\prime}, t\right) G_{l}\left(t, t^{\prime}\right)\right]
\end{aligned}
$$

The Fourier transform of the function $\mathcal{G}_{i j}\left(t, t^{\prime}\right)$ can be calculated in the usual way, yielding

$$
\begin{aligned}
& \mathcal{G}_{i j}^{-+}(\omega)^{+}=2 \pi \rho_{0}^{2}\left[\beta_{i} \beta_{j}^{*} \frac{e V+\omega}{2}\left[-1+\operatorname{coth}\left(\beta \frac{e V+\omega}{2}\right)\right]+\beta_{i}^{*} \beta_{j} \frac{e V-\omega}{2}\left[1+\operatorname{coth}\left(\beta \frac{e V-\omega}{2}\right)\right]\right] \\
& \mathcal{G}_{i j}^{+-}(\omega)^{+}=2 \pi \rho_{0}^{2}\left[\beta_{i} \beta_{j}^{*} \frac{e V+\omega}{2}\left[1+\operatorname{coth}\left(\beta \frac{e V+\omega}{2}\right)\right]+\beta_{i}^{*} \beta_{j} \frac{e V-\omega}{2}\left[-1+\operatorname{coth}\left(\beta \frac{e V-\omega}{2}\right)\right]\right] \\
& \overline{\mathcal{G}}_{i i}^{--}(\omega)=\overline{\mathcal{G}}_{i i}^{++}(\omega)=\beta_{i} \beta_{i}^{*} \int \frac{d \omega_{1}}{2 \pi}\left[G_{r}^{--}\left(\omega_{1}+\omega\right) G_{l}^{--}\left(\omega_{1}\right)-G_{r}^{--}\left(\omega_{1}\right) G_{l}^{--}\left(\omega_{1}+\omega\right)\right] \\
&=2 \pi \rho_{0}^{2} \beta_{i} \beta_{i}^{*} \sigma^{-}(\omega, V),
\end{aligned}
$$

where $\sigma^{-}(\omega, V)$ is given in Eq. 43 . 


\section{Appendix B: Details of the second order current noise calculation}

The starting point for the calculation is Eq. (41), together with Eqs. (11, 12) the symmetrized current noise in the non-stationary case can be written as

$$
\begin{aligned}
S_{\mathrm{sym}}^{(2)}(\omega, & \left.t^{\prime}\right)=\frac{e^{2}}{2} \int d t e^{i \omega t}\left\{\mathcal{G}_{00}^{-+}(t)+\mathcal{G}_{00}^{+-}(t)+\right. \\
+ & \left\langle\hat{x}\left(t^{\prime}\right)\right\rangle\left[\mathcal{G}_{01}^{-+}(t)+\mathcal{G}_{01}^{+-}(t)\right]+\left\langle\hat{x}\left(t^{\prime}\right)\right\rangle \cos (\Omega t)\left[\mathcal{G}_{10}^{-+}(t)+\mathcal{G}_{10}^{-+}(t)\right]+\frac{\left\langle\hat{p}\left(t^{\prime}\right)\right\rangle}{m \Omega} \sin (\Omega t)\left[\mathcal{G}_{10}^{-+}(t)+\mathcal{G}_{10}^{+-}(t)\right]+ \\
+ & \left.\left\langle\hat{x}\left(t^{\prime}\right) \hat{x}\left(t^{\prime}\right)\right\rangle \cos (\Omega t)\left[\mathcal{G}_{11}^{-+}(t)+\mathcal{G}_{11}^{+-}(t)\right]+\frac{\left\langle\hat{x}\left(t^{\prime}\right) \hat{p}\left(t^{\prime}\right)\right\rangle}{m \Omega} \sin (\Omega t) \mathcal{G}_{11}^{-+}(t)+\frac{\left\langle\hat{p}\left(t^{\prime}\right) \hat{x}\left(t^{\prime}\right)\right\rangle}{m \Omega} \sin (\Omega t) \mathcal{G}_{11}^{+-}(t)\right\} .
\end{aligned}
$$

The further calculation is straightforward by using Eq. A4 and Eq. A5. Finally, the current noise $S_{\mathrm{sym}}^{(2)}\left(\omega, t^{\prime}\right)$ in Eq. (B1) can be written as

$$
\begin{aligned}
& S_{\mathrm{sym}}^{(2)}\left(\omega, t^{\prime}\right)=\frac{e^{2}}{2}\left\{\mathcal{G}_{00}^{-+}(\omega)+\mathcal{G}_{00}^{+-}(\omega)+\left\langle\hat{x}\left(t^{\prime}\right)\right\rangle\left[\mathcal{G}_{01}^{-+}(\omega)+\mathcal{G}_{01}^{+-}(\omega)\right]+\right. \\
& +\frac{1}{2}\left\langle\hat{x}\left(t^{\prime}\right)\right\rangle\left[\mathcal{G}_{10}^{-+}(\omega+\Omega)+\mathcal{G}_{10}^{-+}(\omega-\Omega)+\mathcal{G}_{10}^{+-}(\omega+\Omega)+\mathcal{G}_{10}^{+-}(\omega-\Omega)\right]- \\
& -\frac{i}{2 m \Omega}\left\langle\hat{p}\left(t^{\prime}\right)\right\rangle\left[\mathcal{G}_{10}^{-+}(\omega+\Omega)-\mathcal{G}_{10}^{-+}(\omega-\Omega)+\mathcal{G}_{10}^{+-}(\omega+\Omega)-\mathcal{G}_{10}^{+-}(\omega-\Omega)\right]+ \\
& +\frac{1}{2}\left\langle\hat{x}\left(t^{\prime}\right) \hat{x}\left(t^{\prime}\right)\right\rangle\left[\mathcal{G}_{11}^{-+}(\omega+\Omega)+\mathcal{G}_{11}^{-+}(\omega-\Omega)+\mathcal{G}_{11}^{+-}(\omega+\Omega)+\mathcal{G}_{11}^{+-}(\omega-\Omega)\right]- \\
& \left.-\frac{i}{2 m \Omega}\left\langle\hat{x}\left(t^{\prime}\right) \hat{p}\left(t^{\prime}\right)\right\rangle\left[\mathcal{G}_{11}^{-+}(\omega+\Omega)-\mathcal{G}_{11}^{-+}(\omega-\Omega)\right]-\frac{i}{2 m \Omega}\left\langle\hat{p}\left(t^{\prime}\right) \hat{x}\left(t^{\prime}\right)\right\rangle\left[\mathcal{G}_{11}^{+-}(\omega+\Omega)-\mathcal{G}_{11}^{+-}(\omega-\Omega)\right]\right\} .
\end{aligned}
$$

The functions $\sigma^{ \pm}(\xi, V)$, see Eq. 43, allow us to distinguishing the Markovian from the non-Markovian regime. For $T \rightarrow 0$ we find

$$
\begin{aligned}
& \sigma^{-}(\xi, V)= \begin{cases}\operatorname{sgn}(V) \xi & e|V|>\xi \\
\operatorname{sgn}(V) e|V| & e|V|<\xi\end{cases} \\
& \sigma^{+}(\xi, V)= \begin{cases}e|V| & e|V|>\xi \\
\xi & e|V|<\xi\end{cases}
\end{aligned}
$$

where $T$ here is the temperature of electrons in the leads.

\section{Appendix C: Details of the fourth order current noise calculation}

We first give the whole expression for the current noise to fourth order in the tunneling amplitudes containing the $\mathcal{M}$-functions of Eq. (50) and oscillator operators

$$
\begin{aligned}
S^{(4)} & \left(\tau_{3}, \tau_{4}\right)=-\frac{e^{2}}{2} \int_{c} d \tau_{1} d \tau_{2}\{ \\
& \mathcal{M}_{0,0,0,0}\left(\tau_{1}, \tau_{2}, \tau_{3}, \tau_{4}\right)+ \\
& +\mathcal{M}_{0,0,0,1}\left(\tau_{1}, \tau_{2}, \tau_{3}, \tau_{4}\right)\left\langle\hat{x}\left(\tau_{4}\right)\right\rangle+\mathcal{M}_{0,0,1,0}\left(\tau_{1}, \tau_{2}, \tau_{3}, \tau_{4}\right)\left\langle\hat{x}\left(\tau_{3}\right)\right\rangle+ \\
& +\mathcal{M}_{0,1,0,0}\left(\tau_{1}, \tau_{2}, \tau_{3}, \tau_{4}\right)\left\langle\hat{x}\left(\tau_{2}\right)\right\rangle+\mathcal{M}_{1,0,0,0}\left(\tau_{1}, \tau_{2}, \tau_{3}, \tau_{4}\right)\left\langle\hat{x}\left(\tau_{1}\right)\right\rangle+ \\
& +\mathcal{M}_{0,0,1,1}\left(\tau_{1}, \tau_{2}, \tau_{3}, \tau_{4}\right)\left\langle T_{c} \hat{x}\left(\tau_{3}\right) \hat{x}\left(\tau_{4}\right)\right\rangle+\mathcal{M}_{0,1,0,1}\left(\tau_{1}, \tau_{2}, \tau_{3}, \tau_{4}\right)\left\langle T_{c} \hat{x}\left(\tau_{2}\right) \hat{x}\left(\tau_{4}\right)\right\rangle+ \\
& +\mathcal{M}_{0,1,1,0}\left(\tau_{1}, \tau_{2}, \tau_{3}, \tau_{4}\right)\left\langle T_{c} \hat{x}\left(\tau_{2}\right) \hat{x}\left(\tau_{3}\right)\right\rangle+\mathcal{M}_{1,0,0,1}\left(\tau_{1}, \tau_{2}, \tau_{3}, \tau_{4}\right)\left\langle T_{c} \hat{x}\left(\tau_{1}\right) \hat{x}\left(\tau_{4}\right)\right\rangle+ \\
& +\mathcal{M}_{1,0,1,0}\left(\tau_{1}, \tau_{2}, \tau_{3}, \tau_{4}\right)\left\langle T_{c} \hat{x}\left(\tau_{1}\right) \hat{x}\left(\tau_{3}\right)\right\rangle+\mathcal{M}_{1,1,0,0}\left(\tau_{1}, \tau_{2}, \tau_{3}, \tau_{4}\right)\left\langle T_{c} \hat{x}\left(\tau_{1}\right) \hat{x}\left(\tau_{2}\right)\right\rangle+ \\
& +\mathcal{M}_{0,1,1,1}\left(\tau_{1}, \tau_{2}, \tau_{3}, \tau_{4}\right)\left\langle T_{c} \hat{x}\left(\tau_{2}\right) \hat{x}\left(\tau_{3}\right) \hat{x}\left(\tau_{4}\right)\right\rangle+\mathcal{M}_{1,0,1,1}\left(\tau_{1}, \tau_{2}, \tau_{3}, \tau_{4}\right)\left\langle T_{c} \hat{x}\left(\tau_{1}\right) \hat{x}\left(\tau_{3}\right) \hat{x}\left(\tau_{4}\right)\right\rangle+ \\
& +\mathcal{M}_{1,1,0,1}\left(\tau_{1}, \tau_{2}, \tau_{3}, \tau_{4}\right)\left\langle T_{c} \hat{x}\left(\tau_{1}\right) \hat{x}\left(\tau_{2}\right) \hat{x}\left(\tau_{4}\right)\right\rangle+\mathcal{M}_{1,1,1,0}\left(\tau_{1}, \tau_{2}, \tau_{3}, \tau_{4}\right)\left\langle T_{c} \hat{x}\left(\tau_{1}\right) \hat{x}\left(\tau_{2}\right) \hat{x}\left(\tau_{3}\right)\right\rangle+ \\
& \left.+\mathcal{M}_{1,1,1,1}\left(\tau_{1}, \tau_{2}, \tau_{3}, \tau_{4}\right)\left\langle T_{c} \hat{x}\left(\tau_{1}\right) \hat{x}\left(\tau_{2}\right) \hat{x}\left(\tau_{3}\right) \hat{x}\left(\tau_{4}\right)\right\rangle\right\}+\int_{c} d \tau_{1} d \tau_{2}\left\langle T_{c} \hat{H}_{\mathrm{tun}}\left(\tau_{1}\right) \hat{I}\left(\tau_{3}\right)\right\rangle\left\langle T_{c} \hat{H}_{\mathrm{tun}}\left(\tau_{2}\right) \hat{I}\left(\tau_{4}\right)\right\rangle .
\end{aligned}
$$


A first reduction of terms in Eq. (C1) is done by only focusing on the stationary case. This allows us to drop terms which are proportional to $\langle\hat{x}(t)\rangle$ of Eq. (C1) and only keep terms proportional to $D\left(t, t^{\prime}\right)$ and $D\left(t, t^{\prime}\right) D\left(t^{\prime \prime}, t^{\prime \prime \prime}\right)$. Unlinked diagrams which appear in this expression are canceled by the $\hat{I}^{2}$ term which is always of the bubble type.

1 K. Schwab and M. Roukes, Physics Today 58, 36 (2005).

2 A. A. Clerk, S. M. Girvin, F. Marquardt, and R. J. Schoelkopf, Rev. Mod. Phys. 82, 1155 (2010).

3 Y. Yang, C. Callegari, X. Feng, K. Ekinci, and M. Roukes, Nano Lett 6, 583 (2006).

${ }^{4}$ H. Mamin and D. Rugar, Applied Physics Letters 79, 3358 (2001).

5 M. LaHaye, O. Buu, B. Camarota, and K. Schwab, Science 304, 74 (2004).

6 B. Lassagne, Y. Tarakanov, J. Kinaret, D. Garcia-Sanchez, and A. Bachtold, Science 325, 1107 (2009).

7 G. A. Steele, A. K. Huttel, B. Witkamp, M. Poot, H. B. Meerwaldt, L. P. Kouwenhoven, and H. S. J. V. D. Zant, Science 325, 1103 (2009).

8 M. Hofheinz, H. Wang, M. Ansmann, R. C. Bialczak, E. Lucero, M. Neeley, A. D. O'connell, D. Sank, J. Wenner, J. M. Martinis, et al., Nature 459, 546 (2009).

9 A. M. O'Connell, M. Hofheinz, M. Ansmann, R. C. Bialczak, M. Lenander, E. Lucero, M. Neeley, D. Sank, H. Wang, M. Weides, et al., Nature 464, 697 (2010).

10 F. Marquardt, J. Chen, A. Clerk, and S. Girvin, Phys. Rev. Lett. 99, 093902 (2007).

11 I. Wilson-Rae, N. Nooshi, W. Zwerger, and T. Kippenberg, Phys. Rev. Lett. 99, 093901 (2007).

12 T. Rocheleau, T. Ndukum, C. Macklin, J. B. Hertzberg, A. A. Clerk, and K. C. Schwab, Nature 463, 72 (2010).

13 A. Naik, O. Buu, M. LaHaye, A. Armour, A. Clerk, M. Blencowe, and K. Schwab, Nature 443, 193 (2006).
14 A. Clerk and S. Girvin, Phys. Rev. B 70, 121303 (2004).

15 C. Doiron, B. Trauzettel, and C. Bruder, Phys. Rev. B 76, 195312 (2007).

16 C. Doiron, B. Trauzettel, and C. Bruder, Phys. Rev. Lett. 100, 027202 (2008).

17 J. Wabnig, J. Rammer, and A. Shelankov, Phys. Rev. B 75, 205319 (2007).

18 G. Rastelli, M. Houzet, and F. Pistolesi, Eur. Phys. Lett. 89, 57003 (2010).

19 N. Flowers-Jacobs, D. Schmidt, and K. Lehnert, Phys. Rev. Lett. 98, 096804 (2007).

20 T. L. Schmidt, K. Børkje, C. Bruder, and B. Trauzettel, Phys. Rev. Lett. 104, 177205 (2010).

21 J. Rammer and H. Smith, Rev. Mod. Phys. 58, 323 (1986).

22 J. Rammer, Quantum field theory of non-equilibrium states (2007).

23 D. Mozyrsky and I. Martin, Phys. Rev. Lett. 89, 018301 (2002).

24 A. Clerk, Phys. Rev. B 70, 245306 (2004).

25 Y. Blanter and M. Büttiker, Physics Reports 336, 1 (2000).

26 R. Schoelkopf, P. Burke, A. Kozhevnikov, D. Prober, and M. Rooks, Phys. Rev. Lett. 78, 3370 (1997).

27 L. DiCarlo, Y. Zhang, D. McClure, C. Marcus, L. Pfeiffer, and K. West, Review of Scientific Instruments 77, 073906 (2009).

28 J. Eisert, M. B. Plenio, S. Bose, and J. Hartley, Phys. Rev. Lett. 93, 190402 (2004). 